\title{
Correlated disorder in a model binary glass through a local SU(2) bonding topology
}

\author{
P. M. Derlet* \\ Condensed Matter Theory Group, Paul Scherrer Institut, CH-5232 Villigen PSI, Switzerland
}

(Received 16 July 2020; revised 28 October 2020; accepted 24 November 2020; published 14 December 2020)

\begin{abstract}
A quantitative understanding of the microscopic constraints, which underlie a well relaxed glassy structure, is the key to developing a microscopic theory of structural evolution and plasticity for the amorphous metallic solid. Here we demonstrate the applicability of one such theory of local bonding constraints developed by $\mathrm{D}$. R. Nelson [Phys. Rev. B 28, 5515 (1983)] for a model binary Lennard-Jones glass structure. By introducing a modified radical Voronoi tessellation, which removes some ambiguity in how nearest-neighbor bonds are enumerated, it is found, that a large proportion ( $>95 \%$ ) of local atomic environments follow the connectivity rules of the $\mathrm{SU}(2)$ topology resulting in a dense network of disclination lines characterizing the defect bonds. Furthermore, it is numerically shown that a low-energy glass structure corresponds to a reduced level of bond-length frustration and thus a minimally defected bond-defect network. It is then demonstrated that such a defect network provides a framework to analyze thermally activated structural excitations, revealing those high-energy/low-density/elastically soft regions not following the connectivity constraints are more likely to undergo structural rearrangement that often ends with the creation of new SU(2) local topology content. The work provides a new analysis tool to study the connectivity of developing structural motives characteristic of isotropic undercooled liquids, their transition to a glass, and subsequent glassy structural relaxation.
\end{abstract}

DOI: 10.1103/PhysRevMaterials.4.125601

\section{INTRODUCTION}

If the temperature of a glass forming metallic liquid is lowered sufficiently fast below the melting point, the nucleation and growth processes needed for crystallization do not occur and the material enters the metastable undercooled liquid phase. Kauzmann, in his seminal paper of 1948 [1], asserted that as the temperature continues to drop, fluctuation processes rare to the equilibrium liquid become increasingly likely. These microscopic fluctuations, broadly known as dynamical heterogenities [2,3], have characteristic timescales and length-scales which rapidly increase with decreasing temperature and when these become larger than the relevant observational timescales, the material falls out of the metaequilibrium of the undercooled liquid and enters that of the bulk-metallic glass $[1,4,5]$.

The materials science approach is to quantify the nature of this glassy metaequilibrium regime-its structural characteristics through its fluctuations and instabilities, and ultimately their collective and macroscopic response to stimuli such as external heating and/or loading protocols [6-8]. With no long-range order, understanding amorphous structure has to be based on quantifying the atomic-scale constraints. Indeed, the configurational entropies of a glass can be up to an order of magnitude lower than its liquid phase at the melting temperature resulting in a considerable reduction of the accessible structural phase space [5]. The classification of such constraints, and how they may be broken, can lead to the

\footnotetext{
*Peter.Derlet@psi.ch
}

notion of a structural defect hierarchy which in turn could be the basis for a microscopic theory of the amorphous solid.

Kauzmann goes on to assert that deep within the undercooled liquid those microscopic structural fluctuations necessary for crystal nucleation, and those deemed to be rare in the equilibrium liquid state, have comparable timescales, competing with each other to determine the metaequilibrium structure of the undercooled liquid, and therefore that of the resulting glass structure. This suggests that the sought after atomic-scale constraints of the glass feature aspects of local structural motives compatible with local crystalline order $[9,10]$.

There has been considerable effort to characterize within atomistic simulation, the local structural motives of the glass and more generally that of the undercooled liquid, and the glass transition it entails (see, for example, the review article of Tanaka and co-workers [11], which considers both isotropic and molecular-type liquids). Such approaches address both static structures and their dynamics, thus broadly addressing both local entropic and energetic aspects of the problem. This latter perspective seems intuitive since as the temperature lowers, entropic factors diminish and the energetics of the local motives begin to dominate. It is within this "low-energy" sector that the aforementioned constraints should emerge.

Insight into those structural fluctuations that are not compatible with general crystalline order, and which are rare in the liquid, can be gained by considering the minimum energy configuration of a quadruple of atoms [6]. For a monoatomic system, this configuration results in a regular tetrahedron. However, such regular tetrahedra can not be packed in a volume filling way. The packing of twelve distorted tetrahedrons around a single atom, forms an icosahedron, and represents 

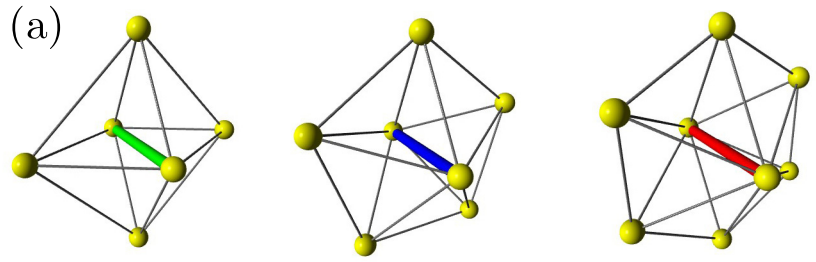

(b)

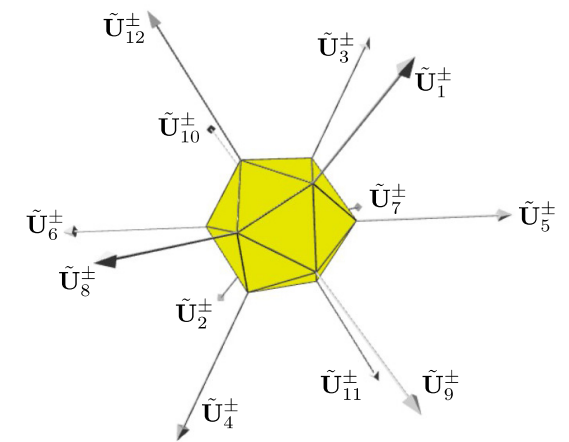

(c)
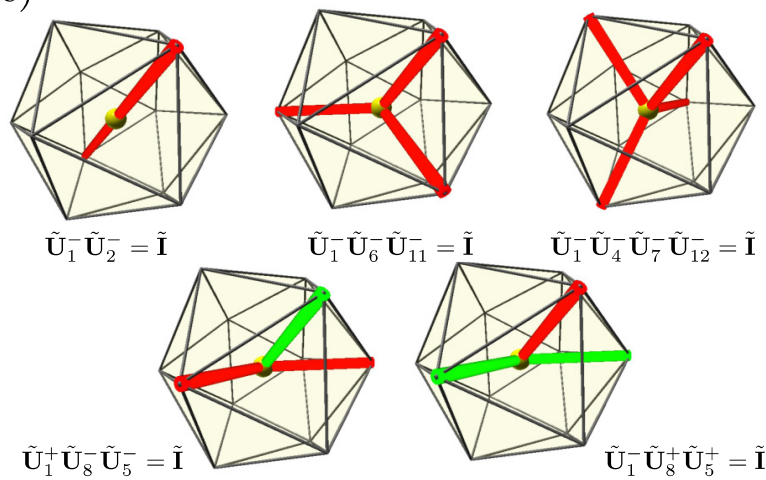

FIG. 1. (a) Four (green), five (blue), and six (red) fold bonds, defined by the number of common neighbors or the number of tetrahedra that may be packed around the bond. The creation of a defect involves the breaking of the fivefold (middle) symmetry to that of either the fourfold (left) or the sixfold (right) symmetry. (b) Yellow shaded icosahedron whose fivefold rotation symmetry directions are labeled from $i=1$ to 12 . To each such axis corresponds the operator, $\tilde{\mathbf{U}}_{i}^{ \pm}$, which creates a fourfold (+) or a sixfold (-) bond defect that breaks the corresponding fivefold symmetry along that direction. The six fivefold rotation axes correspond to the $\tilde{\mathbf{U}}_{1}^{ \pm}=\tilde{\mathbf{U}}_{2}^{\mp}, \tilde{\mathbf{U}}_{3}^{ \pm}=$ $\tilde{\mathbf{U}}_{4}^{\mp}, \tilde{\mathbf{U}}_{5}^{ \pm}=\tilde{\mathbf{U}}_{6}^{\mp}, \tilde{\mathbf{U}}_{7}^{ \pm}=\tilde{\mathbf{U}}_{8}^{\mp}, \tilde{\mathbf{U}}_{9}^{ \pm}=\tilde{\mathbf{U}}_{10}^{\mp}$, and $\tilde{\mathbf{U}}_{11}^{ \pm}=\tilde{\mathbf{U}}_{12}^{\mp}$ redundancies. (c) Examples of allowed bond defects where green/red defect bonds represent fourfold/sixfold bonds, using the same orientation as in (b). Here the upper panels represent examples of Frank-Kasper polyhedra [14] and the lower panels examples of Nelson polyhedra $[29,30]$.

the minimally distorted tetrahedral packing with the bond to the central atom being shorter than that between its neighbors. The common neighbors between the central atom and one of its neighbors, number five, and represent a packing of five tetrahedra. This is referred to as a fivefold bond [Fig. 1(a)]. Continuing a tetrahedral packing protocol, whilst maintaining the icosahedral point symmetry soon results in distorted tetrahedra and strong internal strain, that can be alleviated by either modifying the curvature of the space in which the particles are embedded $[12,13]$ or by breaking the icosahedral point symmetry and introducing "defects" in the form of fourfold and sixfold bonds-an idea proposed by Frank and
Kasper [14] where generally an $n$-fold bond is represented by the packing of $n$ distorted tetrahedra around the bond axis [Fig. 1(a)]. It is these fourfold and sixfold bonds that lead to long range close-packed crystalline order, resulting in these bonds being referred to as "crystal-like" bonds, whereas the fivefold bond is referred to as the "liquidlike" bond (which, in fact, is rare in the equilibrium liquid). Relative to the Z12 coordinated local structure of the icosahedral environment, Frank and Kasper [14] identified these bonds as higher coordinated environments involving two, three, four additional sixfold bonds whose coordinations are, respectively, 13, 14, and 15, whereas Bernal [15] identified lower coordinated environments as involving two, three, and four additional fourfold bonds whose coordinations are, respectively, Z11, Z10, and Z9.

The discussed structural motives are all relevant for the amorphous structure, as evidenced by the success of the glass structural models of Miracle and co-workers [16-19], and the use of icosahedral content and the polytope variants pioneered by Ma and co-workers [20-22]. The work of Miracle demonstrates that structural insight into binary and ternary metallic glasses can be gained through an analysis of dense local packings of solute atoms which take into account the coordination of the neighboring atoms [16], whilst the work of Ma demonstrates the central importance of local icoshadral environments as a structural measure of relaxation and rejuvenation in bulk metallic glasses. Indeed, Jónsson and Andersen [23] had showed somewhat earlier, that as the glass-transition temperature regime is entered and passed, a local icosahedral ordering rapidly percolates throughout the model Lennard Jones amorphous solid. Together, these and many subsequent works introduce the notion of medium range order, through the connectivity of how such local structural units may be packed.

Atomistic simulation of well relaxed model binary alloys has given some insight into the nature of such medium range order. The work of Zemp et al. [24,25] has demonstrated for a model $\mathrm{CuZu}$ glass, that high temperature annealing simulations spanning up to $800 \mathrm{nsec}$ result in atomic environments whose icosahedrally coordinated atoms form local bond networks identified as fragments of the C15 Laves phase - a crystal structure belonging to the class of closepacked topological alloy structures which are dominated by fivefold and sixfold bonds. This has also been observed using the Wahnström [26] Lennard Jones potential [9,27]—a potential that is known to nucleate Laves crystals at high enough temperatures [28].

The notion of fourfold and sixfold bonds being defected bonds was put on a firm theoretical founding by Nelson $[29,30]$. Central to this approach was the focus on the geometrical frustration associated with the inability to simultaneously minimize the energy of all bonds in three spatial dimensions [6]. Here, the fivefold bond and its packing of five distorted tetraherons represents a locally minimally frustrated structure at the individual bond-length scale, whereas the icosahedral environment of twelve fivefold bonds represents the minimally frustrated structure of the nearest-neighbor environment length-scale. Inspired by homotopy theory [31], Nelson demonstrated that fourfold and sixbold bond defects at this latter length scale are restricted to a subset of nearestneighbor environments-see Sec. II. The connectivity of these 
restricted local topologies could then represent a robust mathematical representation of the amorphous structure, defining the glassy structure in terms of microscopic constraints that the local atomic environments must satisfy in which any low-energy microscopic realization would be described by a network of $n(\neq 5)$-fold bond defect lines-the so-called four- and sixfold disclination line defects. Work by Qi and Wang [32], who studied a 500 atom model $\mathrm{Mg}_{3} \mathrm{Ca}_{7}$ system found several realizations of the local topologies predicted by Nelson, in terms of the number of four-, five-, and sixfold bond number, confirming that such local structural motives do exist.

It is the goal of the present work to quantitatively investigate to what degree the above disclination network description is applicable to glassy structures obtained through atomistic simulation, a framework which gives predictions on the allowed bonding structure not only in terms of the $n$-fold character but also its allowed orientational geometry. This is done for the Wahnström [26] Lennard Jones model binary glass, for glassy structures produced directly from the undercooled melt and also from subsequent isothermal evolution at timescales in the micro-second range. It is found that a large percentage of the glassy structure, approximately $95 \%$ for well relaxed systems, do indeed follow the topological constraints, producing a complex system spanning disclination network. In doing so, it is established that glass relaxation entails a reduction of frustration corresponding to an increasingly less dense disclination network.

The paper is organized as follows. In Sec. II, the relevant aspects of Nelson's work are introduced leading to a mathematical theory of local constraints based on the SU(2) algebra. The results section, Sec. III, begins with a description of the atomistic model binary glass simulations to which the SU(2) algebra is applied, and a modified radical Voronoi tessellation is developed that is better able to define the local nearestneighbor geometry necessary to define the $n$-fold bond defect structure of the produced microscopic samples. This modification is developed within the OVITO visualization software [33] and made publicly available via GitHub [34]. Here one will also find a stand alone fortran90 implementation that uses the open source VORO++ TESSELLATION software [35]. This is followed by application of the SU(2) algebra to these glassy structures (Secs. III A and III B), and how it correlates with traditional microscopic descriptors such as local atomic volume (free volume), local energy, local stress, frustration, and elastic stiffness (Sec. III C). Sec. III D investigates the spatial properties of the disclination network. Section IV discusses how the present work relates to the efficient packing glass-structure model of Miracle and co-workers [16-19] and how the SU(2) framework allows for an entirely new aspect to studying thermally activated localized structural excitations, and how they can lead to structural relaxation with the creation of minimally defected content. The results are also discussed within the context of past coarse-graining approaches that take into account the constraints imposed by the SU(2) algebra and also contemporary dynamical facilitation theories of the glass transition. Section V summarizes the work and discusses future directions. The paper will refer extensively to the supplementary materials document [36], where corresponding section and figure numbers will be prefixed with SM.

\section{THE SU(2) ALGEBRA OF LOCAL STRUCTURE}

Nelson begins with the flat defect-free 2D triangular lattice whose atoms are Z' 6 coordinated. The Z' terminology refers to nearest-neighbor coordinations in $2 \mathrm{D}$. The associated Z'5 and Z'7 local coordination defect environments are topological (referred to as disclinations) and maybe associated with the homotopy group arising from the translational and rotational symmetry of the liquid phase in two dimensions, and the discrete lattice symmetry of the solid 2D triangular phase. This $2 \mathrm{D}$ picture is then applied to the surface of a 3 -sphere where the discrete lattice symmetry is replaced with the discrete symmetries of the icosahedron-the curved space and finite 2D triangular lattice analog. The defect free atomic structure would represent the environment of the Z12 coordinated icosahedron structure of an atom within a 3D glass, with a surface defect-free coordination of Z'5. Surface defect coordinations of Z' 4 and Z' 6 are now the fourfold and sixfold bonds referred to earlier. See Fig. 1.

In what follows only the fivefold symmetry of the icosahedron are considered, and in particular the conjugacy classes associated with \pm 72 rotations around the $6+6$ fivefold axes (see Sec. IIC in Ref. [30]) which may be identified as the fundamental disclinations of the icosahedron solid. The corresponding group elements of these defect types forms an SU(2) algebra, defined by the matrices:

$$
\tilde{\mathbf{U}}_{i}^{ \pm}=\exp \left(\frac{1}{2} i \omega^{ \pm} \mathbf{n}_{i} \cdot \tilde{\boldsymbol{\sigma}}\right),
$$

where $\mathbf{n}_{i}$ are the twelve fivefold rotation axes of the icosahedron [see Fig. 1(b)], $\omega^{+}=72^{\circ}$ and $\omega^{-}=-72^{\circ}$ refer to the so-called disclinations associated with, respectively, the fourand sixfold bonds, and $\sigma$ is the 3-vector of Pauli matrices. The allowed $n$-fold bond structure of a particular atom is then defined by products of the $\tilde{\mathbf{U}}_{i}^{ \pm}$giving the identity matrix. For example the Frank-Kasper Z14 polyhedron [14], could be given by $\tilde{\mathbf{U}}_{1}^{-} \tilde{\mathbf{U}}_{2}^{-}=\tilde{\mathbf{I}}$, the Frank-Kasper Z15 polyhedron by $\tilde{\mathbf{U}}_{1}^{-} \tilde{\mathbf{U}}_{5}^{-} \tilde{\mathbf{U}}_{11}^{-}=\tilde{\mathbf{I}}$, and the Frank-Kasper Z16 polyhedron by $\tilde{\mathbf{U}}_{1}^{-} \tilde{\mathbf{U}}_{8}^{-} \tilde{\mathbf{U}}_{9}^{-} \tilde{\mathbf{U}}_{12}^{-}=\tilde{\mathbf{I}}$ matrix product. The Bernal-hole bond order structures [15] are given by similar products, but in terms of the $\mathbf{U}_{i}^{+}$. Reference [30] also introduces the so-called vacancy (free-volume defect) in which one sixfold bond is created and two 4-bonds are created, as well as interstitial (negative free-volume defect) in which one fourfold bond and two sixfold bonds are created at a site. These polyhedra will be referred to as Z11 and Z13 Nelson polyhedra. Algebraically these take the respective forms of $\tilde{\mathbf{U}}_{1}^{-} \tilde{\mathbf{U}}_{5}^{+} \tilde{\mathbf{U}}_{12}^{-}=\tilde{\mathbf{I}}$ and $\tilde{\mathbf{U}}_{1}^{+} \tilde{\mathbf{U}}_{6}^{+} \tilde{\mathbf{U}}_{9}^{-}=\tilde{\mathbf{I}}$. The Frank-Kasper and Nelson polyhedra are visualized in terms of the geometry entailed by Eq. (1) in Fig. 1(c). There are many more allowed defected bond order structures, some of which will be studied in more detail in what follows.

The implication for connectivity of a defected bond-order network satisfying this algebra throughout the amorphous structure is clear. A network of disclination lines whose interconnectivity satisfies the local need for the product of the correspond matrices to give the identity operator. As pointed out by Nelson, an immediate consequence is that a fourfold or sixfold bond, disclination line, cannot terminate at a site that is otherwise defect free since obviously, $\tilde{\mathbf{U}}_{i}^{ \pm} \neq \tilde{\mathbf{I}}$. 


\section{APPLICATION TO A MODEL BINARY GLASS AND A MODIFIED RADICAL VORONOI TESSELLATION}

For the present work, the model binary Lennard-Jones potential of Wahnström [26] is used. This potential is well known as a model fragile glass former [13,37-39] and is able to capture the essential structural physics of bulk binary metallic glasses such as that of $\mathrm{CuZr}[6,20,22,40]$. For the current work four, 32000 atom, atomic configurations are considered. Each has a 50:50 stoichiometry of small to large atoms which is close to the potential's eutectic composition. Periodic boundary conditions are used. Glass structures are produced from the melt using a protocol of quenching and iso-thermal annealing. The precise details are given in the Secs. SM I and SM II. One configuration is referred to as "from-the-melt" and is produced from a conjugant gradient (cg) quench of a high temperature liquid configuration at $T=1.7 T_{\mathrm{f}}$ ( $T_{\mathrm{f}}$ is the fictive transition temperature), placing the configuration at a local potential energy minimum-a socalled inherent state $[41,42]$. This configuration may be seen as an extremely unrelaxed glassy structure. The other three samples are obtained from a subsequent isothermal NVT annealing protocol at $T=0.95 T_{\mathrm{f}}$ and are distinguished by their annealing times 0,5 , and $10 \mu \mathrm{sec}$. For structural analysis, the instantaneous atomic configurations are also cg-quenched to remove the thermal noise. All molecular dynamics (MD) simulations are performed using the LAMMPS software platform [43] and atomistic visualization is performed using OVITO [33].

To determine the population of bonds within a given structure, a variant of the Voronoi tesselation [44-46] is used for systems with differently sized atoms. The radical Voronoi tesselation [47] (RV) gives the nearest neighbors of each atom in a polydispersed system, which can be then used to determine the common nearest neighbors of two neighboring atoms. It is assumed that such a radical Voronoi tessellation takes into account all relevant atom type dependencies. This is a working assumption. From this, the $n$-fold nature of each bond is determined via the number of common nearest neighbors the two atoms, forming the bond, have. This approach is motivated by the common neighbor method of Honeycutt and Anderson [48]. It is noted that the number of edges the corresponding Voronoi face has, has also been used to define the $n$-fold bond nature. However, the obtained value is generally not equal to the explicit common neighbor approach, tending to overestimate the value of $n$ due to short edge contributions of more distant (non-nearest-neighbor) atoms.

A modified RV (modRV) is developed to overcome this discrepancy, resulting in Voronoi polyhedra faces whose edge number better corresponds to the number of common neighbors of the associated bond (for details and a python implementation within OVITO [33], see Secs. SM III and SM IV). The modification may be viewed as producing a better tetrahedral tessellation in terms of increasing the regularity [49] of the resulting tetrahedra. In particular, from the standard Voronoi tessellation, $n$ common neighbors of a nearest-neighbor pair can have three neighbors, which among themselves are common neighbors. In terms of a teterahedral tessellation, this results in three highly distorted tetrahedra. The modified radical Voronoi tessellation removes this $n$ - fold bond, resulting in a transition to two less distorted tetrahedra-see Fig. SM 2. This modification generally applies to $n>$ sixfold bonds. Doing so also has the added effect of the resulting tetrahedral tessellation becoming space filling. This modification should be distinguished from a previous modification by Malin and co-workers [50] that also removes more distant neighbors resulting from the standard Voronoi tessellation. The statistical study of the $n$-fold populations has been referred to as the topological $N$-ring analysis [11]. In the following two sections, such statistics will be investigated with the new emphasis on its connectivity according to the work of Nelson [29,30].

\section{A. Investigation of local $\boldsymbol{n}$-fold bond structure}

For each atom, the notation $\left(N_{4}, N_{5}, N_{6}\right)$ is now used. Here $N_{n}$ is the number of $n$-fold bonds. The coordination $Z$ is then given by the sum of the $N_{n}$. This notation should be distinguished from that used to describe a general polytope associated with a (in our case, modified radical) Voronoi tessellation [21,22]. Here, $\left\langle n_{3}, n_{4}, n_{5}, n_{6}, \ldots\right\rangle$ represents a polytope in which $n_{i}$ faces are constructed from $i$ edges, where the labeling $\langle 0,0,12,0, \ldots\rangle$ corresponds to the icosahedron. The presently introduced notation labels the icosahedron as $(0,12,0)$. Atoms with $(0,12, Z-12)$ correspond to $Z>12$-coordinated Frank-Kasper polyhedra, whilst $(2,8,0),(3,6,0)$, and $(4,4,0)$ correspond to the Z10, Z9, and Z8 Bernal holes. The Z13 and Z11 Nelson polyhedra correspond to $(1,10,8)$ and $(2,8,1)$.

Figure 2(a) plots the $n$-fold bond distribution for the four samples resulting from the modRV and standard RV. Figure SM 3 plots the same data using a vertical logarithmic scale. The figures demonstrate that the four-, five-, and sixfold populations dominate using both the modRV and RV tessellations. For the modRV tessellation this dominance is almost complete constituting approximately $99.75 \%$ of the identified bonds. The dominance is somewhat reduced to approximately $98 \%$ when using the standard RV tessellation.

Figure 2(a) demonstrates that the number of defect free fivefold bonds increases whereas the number of fourfold and sixfold defected bonds decrease as the glassy structure relaxes. These trends are compatible with the observed increase in $(0,12,0)$ content seen in Fig. SM $1(b)$ as the glass relaxes. The less pronounced change in $n$-fold bond populations when compared to the scale of icosahedral content change indicates that the creation of such low-energy and high-density icosahedral environments is due to the removal/addition of just a few defect/undefected bonds. Indeed, the $n$-fold bond populations linearly correlate with the icosahedral fractional content, with the gradients equaling $-0.26,0.56$, and -0.29 respectively for the four-, five-, and sixfold bonds. This indicates that the structural transition associated with the creation of icosahedral content involves (on average) the creation of one fivefold bond at the expense of a fourfold and sixfold defected bond. How this may occur will be discussed in Sec. IV. The excess of sixfold bonds over fourfold bonds seen in Fig. 1(a) reflects the dominance of overcoordinated rather than undercoordinated atomic environments and the presence of the Frank-Kasper topologies. More fundamentally this is due to the sixfold bond being less frustrated than the fourfold bond. At the higher 


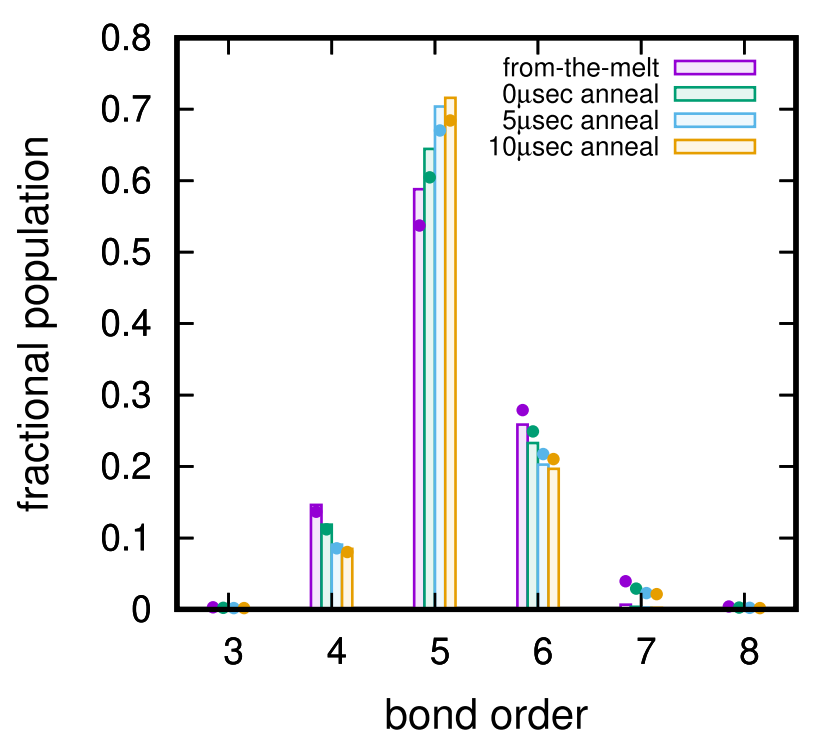

(a)

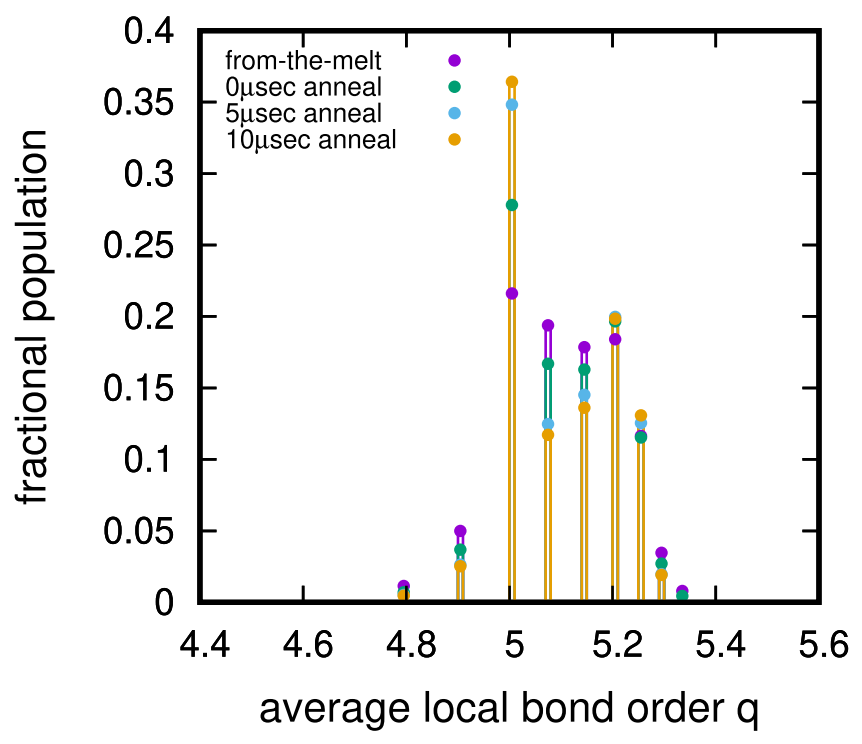

(b)

FIG. 2. Distribution of (a) bond order and (b) local average bond order derived from the from-the-melt, 0,5 , and $10 \mu \mathrm{sec}$ anneal configurations using the modRV tessellation. In (a), data points are also included using the standard RV tessellation.

temperatures of the undercooled liquid this excess is reduced [51].

Figure 3 now enumerates the population fractions of those atomic environments completely defined by the $\left(N_{4}, N_{5}, N_{6}\right)$ terminology, for the $0-, 5-$, and $10-\mu$ s anneal. Only nonzero populations are shown, demonstrating that the horizontal axes satisfies $\left(N_{4}, N_{5}, N_{6}\right)=\left(N_{4}, 12-2 N_{4}, N_{6}\right)$. This originates from the definition $Z=N_{4}+N_{5}+N_{6}$ and the rule $Z=$ $12-N_{4}+N_{6}$. The Frank-Kasper, Bernal-hole, and Nelson polyhedra all satisfy this latter rule, as well as all polyhedra satisfying the $\mathrm{SU}(2)$ algebra and originates from the assertion that non-12 coordinated local environments also apply to the SU(2) algebra in which the creation of a sixfold bond adds an atom to the local environment, and a fourfold bond removes an atom from the local environment [30]. There exists an exception to the above rule. The topology $(1,10,1)$ satisfies $\left(N_{4}, N_{5}, N_{6}\right)=\left(N_{4}, 12-2 N_{4}, N_{6}\right)$ but is not allowed by the $\mathrm{SU}(2)$ algebra of Sec. II since (say) $\tilde{\mathbf{U}}_{1}^{-} \tilde{\mathbf{U}}_{2}^{+} \neq \tilde{\mathbf{I}}$, which indicates that it is not possible to terminate a single fourfold and a single sixfold defect line at an atomic environment containing no other defect lines.

Those local environments that could not be classified in terms of a bond topology are grouped into the "other" classification. For the from-the-melt sample, this is approximately $15 \%$ of the atomic environments, reducing to below $10 \%$ for isothermally annealed samples. Thus as the glassy structure becomes more relaxed an increasingly large percentage of local atomic environments satisfy the aforementioned bonding topologies.

Figure 3 reveals that with increasing relaxation, not only do the $(0,12,0)$ icosahedral environments, but also the Frank-Kasper environments increase in number. The Nelson environments involving a single fourfold bond decrease in number for one and two sixfold bonds and increase for three and four sixfold bonds. All other Nelson environments generally decrease in number. Those environments that do not fit into the $\mathrm{SU}(2)$ connectivity picture also decrease in number as the structure becomes more relaxed. Thus generally, a more relaxed structure entails a reduced number of local environments in which many defect bonds connect. Figure 3 demonstrates that detailed insight into the overall SU(2) structure can also be obtained for relatively unrelaxed glassy structures, with large changes in $\mathrm{SU}(2)$ populations being minimal when much longer relaxation timescales are simulated. Finally, it is noted that using the modRV tessellation, generally results in only a small change in the relative populations of the $\mathrm{SU}(2)$ topologies.

It has to be emphasized that the rule $\left(N_{4}, N_{5}, N_{6}\right)=$ $\left(N_{4}, 12-2 N_{4}, N_{6}\right)$ is a realization of the Euler theorem relating the number of faces (bonds) to the number of edges (sum of bond order over bonds) to the number of vertices of the associated modRV polyhedra. This may be revealed through the average number of edges per face via the average bond order defined as

$$
\bar{q}=\frac{\sum_{n} N_{n} \times n}{\sum_{n} N_{n}},
$$

where $N_{n}$ is the total number of $n$-fold bonds within a given sample. Via Euler's theorem this number can be related to the average coordination via

$$
\bar{Z}=\frac{12}{6-\bar{q}},
$$

indicating that coordination correspondingly reduces with decreasing bond order. Table I displays the common neighbor measured value of $\bar{q}$ and the corresponding prediction for the mean coordination using the above equation for the considered configurations. Also shown is the measured mean coordination derived from the number of faces of each atom's Voronoi polyhedron. Data are shown for both the standard RV and modRV, revealing that the modified RV tessellation results in very good agreement with the predictions of Euler. It is noted that when the number of edges of the common face is 


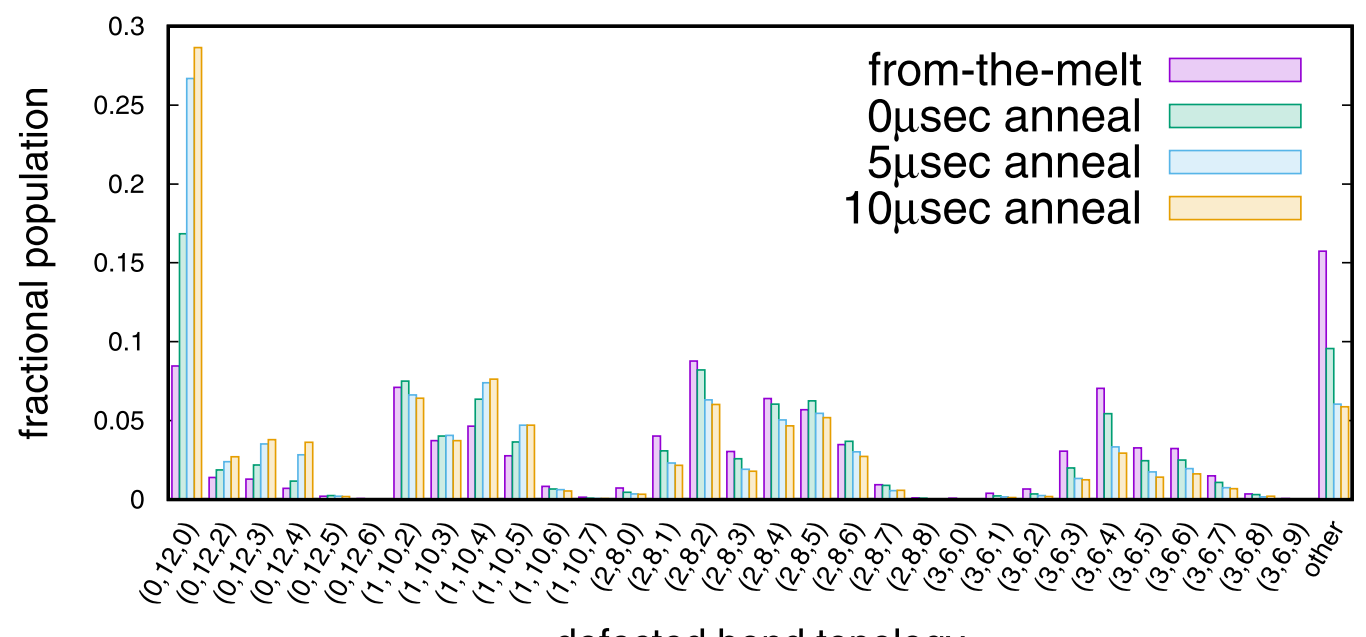

\section{defected bond topology}

FIG. 3. Histogram of local defected bond structures classed as those satisfying $\left(N_{4}, N_{5}, N_{6}\right)=\left(N_{4}, 12-2 N_{4}, N_{6}\right)$ for the from-the-melt, $0-, 5-$, and $10-\mu \mathrm{sec}$ anneal samples. Local environments which do not satisfy this are classed as "other" and will generally involve non-zero values of $N_{7}$.

used to determine the bond order, the Euler relation [Eq. (3)] is always followed, with $\bar{q}$. This emphasizes the modification ensures the correct polyehedra connectivity for the common neighbor definition of bond order. Table I demonstrates that the mean coordination reduces as the glassy structure relaxes towards the value associated with the Frank-Kasper crystal phases $\left(q_{\mathrm{FK}} \approx 5.1\right)$.

The values of $q$ shown in Table I are global averages. Figure 2(b) plots corresponding histograms of the local atomic mean bond order derived from the modRV tessellation, showing a relatively broad but singular distribution. When using the standard RV, a similar overall shape is seen however smaller peaks are seen between the large peaks of the modRV data. The form of Fig. 2(b) can be understood by considering the SU(2) bonding topology populations shown in Fig. 3 and the fact that for a given topology $\left(N_{4}, N_{5}, N_{6}\right)$, the local atomic bond order is $\left(4 N_{4}+5 N_{5}+6 N_{6}\right) /\left(N_{4}+N_{5}+N_{6}\right)$. This value is plotted as a function of $\left(N_{4}, N_{5}, N_{6}\right)$ in Fig. SM 5(a). For the from-the-melt configuration, the distribution has a broad maximum spanning $q$ values between 5 and 5.2, however as the structure relaxes a clear peak at 5 emerges indicating

TABLE I. Calculated average bond order, $\bar{q}$, derived from the common neighbors of a bond, and the average coordination for samples from-the-melt and the 0-, 5-, and 10- $\mu$ sec annealed configurations, using the radical Voronoi tessellation and the modified radical Voronoi tessellation introduced in Sec. SM III.

\begin{tabular}{lccc}
\hline \hline & measured $\bar{q}$ & $\bar{Z}$ [Eq. (3)] & measured $\bar{Z}$ \\
\hline from-the-melt (RV) & 5.229 & 15.567 & 13.958 \\
$0 \mu$ sec (RV) & 5.200 & 15.005 & 13.872 \\
$5 \mu$ sec (RV) & 5.181 & 14.653 & 13.720 \\
$10 \mu$ sec (RV) & 5.175 & 14.553 & 13.762 \\
from-the-melt (modRV) & 5.126 & 13.728 & 13.729 \\
$0 \mu$ sec (modRV) & 5.122 & 13.661 & 13.665 \\
$5 \mu$ sec (modRV) & 5.117 & 13.597 & 13.599 \\
$10 \mu$ sec (modRV) & 5.117 & 13.583 & 13.584 \\
\hline \hline
\end{tabular}

the strong presence of the $(0,12,0)$ icosahedral environment. $(2,8,2)$ environments also contribute to this peak. The peak at 5.2 also increases, reflecting increased Frank-Kasper $(0,12,3)$ and Nelson $(1,10,4)$ content. More generally, the observed reduction in global $\bar{q}$ value with respect to relaxation can be understood as an increase in $(0,12,0)$, an increase Frank-Kasper topologies and some, $N_{4}=1$, Nelson topologies (which push the value towards 5.2), and finally a reduction in $N_{4}=2,3, \ldots$ Nelson topologies (which have average bond orders below 5). Figure SM 5(b) visualizes the glass configurations with atoms colored according to their local $q-$ see Sec. SM VI for a related discussion.

\section{B. Application of SU(2) algebra}

Whilst the results of the previous section strongly support the notion that the $\mathrm{SU}(2)$ bonding rules are largely satisfied in our model binary glass, a quantitative geometric evaluation is still lacking. Figure 1 demonstrates that not only do their exist rules associated with the $\left(N_{4}, N_{5}, N_{6}\right)=\left(N_{4}, 12-2 N_{4}, N_{6}\right)$ classification, there exist relative orientations between the defect bonds. To quantitatively determine to what extent these very specific $n$-fold bond geometries follow the predictions of the SU(2) algebra [Eq. (1) and Fig. 1], the local environment of each $\left(N_{4}, 12-2 N_{4}, N_{6}\right)$ is now investigated in detail.

For each atom identified by $\left(N_{4}, N_{5}, N_{6}\right)$, the following procedure is performed.

(1) The $N_{4}+N_{6}$ defect bond vectors are identified and normalized.

(2) An icosahedron is centered on the central atom and orientated to optimize the alignment of its six fivefold symmetry axes with these bond directions.

(3) Those icosahedron axes closest to the $N_{4}+N_{6}$ bonds are identified with the appropriate $\tilde{\mathbf{U}}_{i}^{ \pm}$operator.

(4) Products of these $N_{4}+N_{6}$ operators which are equal to the identity operator are found to establish that the $\mathrm{SU}(2)$ description is obeyed.

The above procedure identifies the SU(2) bond geometry which needs to be minimally distorted to achieve the actual 


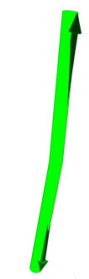

$(2,8,0)$

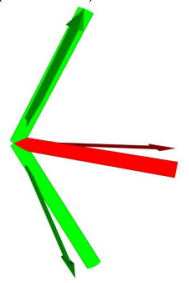

$(2,8,1)$

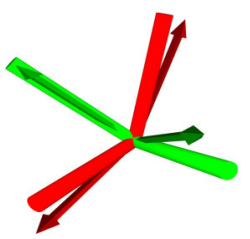

$(2,8,2)$

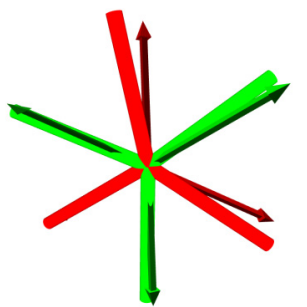

$(3,6,3)$

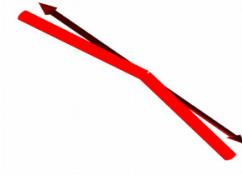

$(0,12,2)$

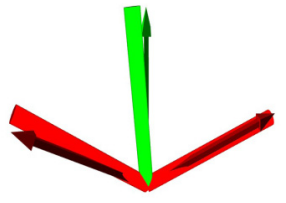

$(1,10,2)$

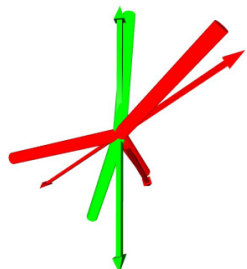

$(2,8,3)$

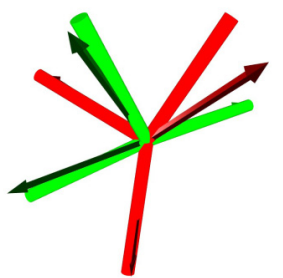

$(3,6,3)$

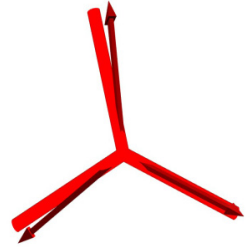

$(0,12,3)$

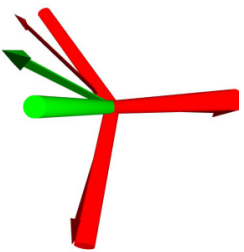

$(1,10,3)$

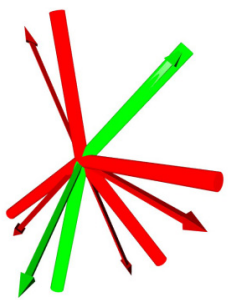

$(2,8,4)$

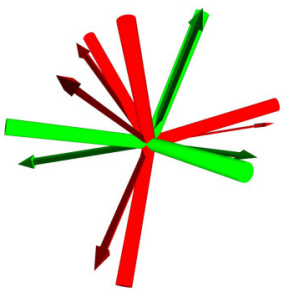

$(3,6,4)$

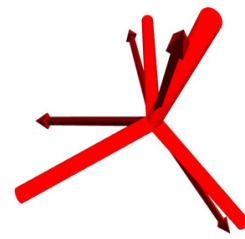

$(0,12,4)$

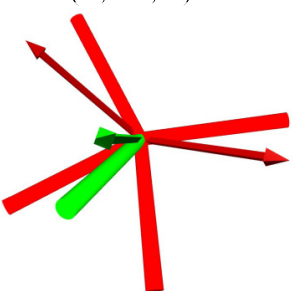

$(1,10,4)$

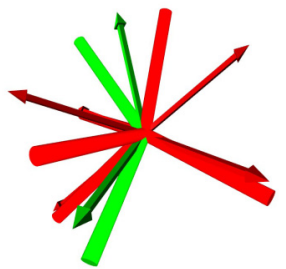

$(2,8,5)$

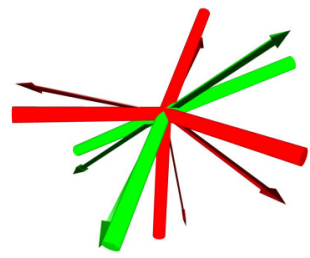

$(3,6,4)$

FIG. 4. Common local defected bond environments derived from the atomistic simulation of a model binary glass. In each panel, the green and red tubes represent the fourfold and sixfold atomic bond vectors, whereas the similarly colored arrows represent the bond vectors associated with to the SU(2) algebra description of Nelson [29,30]. The upper most row show examples of the Z10 Bernal hole, and the Z14-Z16 Frank-Kasper coordinated polyhedra, whereas the second row show examples of the Z11, Z13-Z15 Nelson coordinated polyhedra. The remaining panels show other commonly occurring defected bond geometries associated with the SU(2) algebra.

local atomic structure. Application of the procedure to our glass configuration confirms that all $\left(N_{4}, N_{5}, N_{6}\right)$ topologies identified in Sec. III A do indeed follow the geometry associated with the SU(2) algebra of Sec. II.

Figure 4 visualizes some of the more common nonfive-fold (defected) bonds for a number of these polyhedra obtained from our most relaxed structure. In the figure, the actual bonds (normalized in length) are visualized as green (fourfold) and red (sixfold) tubes, whereas the corresponding fivefold symmetry axes of the rotated icosahedron are visualized as similarly colored normalized vectors. In general, correspondence is excellent for the well known bond geometries. It is emphasized that the $\mathrm{SU}(2)$ description (the precise angular bond geometry associated with the fivefold symmetry axes of the icosahedron) is unable do describe local atomic distortions arising from the interaction between atoms of different type - a certain degree of distortion is thus expected. Both the choice of the orientation (step 2) and the assignment of operators to the bonds (step 3) minimize this distortion by minimizing the angular difference between the atomic bond vectors and the fivefold symmetry axes of the icosahedron. The presence of such distortion is best demonstrated for the case of the $(0,12,4)$ Frank-Kasper defected bond geometry (upper right hand panel of Fig. 4) where the four sixfold bonds arising from the atomic structure exhibit an approximate tetrahedral geometry, a configuration that the fivefold symmetry axes of the icosahedron cannot describe. To quantify the presence of this distortion, the average distortion defined by the angle between the defect bond obtained from simulation and the relevant fivefold symmetry axis of the appropriately orientated icosahedron is calculated. Since the minimal angle between fivefold symmetry axes is approximately $60^{\circ}$, the data show that the distortion is small where, for environments with 1-3 defect bonds the average distortion is between $5^{\circ}$ and $10^{\circ}$. This increases with the number of defect bonds, saturating at appoximately $15^{\circ}$ for greater than five defect bonds. 
These results are summarized in Fig. SM 4 by showing the average angular distortion for each SU(2) topology and also a scatter plot between this average distortion and the fractional populations of $\mathrm{SU}(2)$ topological environments. This latter data demonstrate little correlation between the occurrence and distortion of a particular $\mathrm{SU}(2)$ topology, giving additional indication that the populations within the glassy structure are indeed a result of geometrical constraints rather than say local energetics associated with this distortion.

The results of the present section therefore establish that atomistic simulations produce a model binary amorphous structure which generally follows the connectivity rules as well as the spatial geometry entailed by the $\mathrm{SU}(2)$ algebra formalism of Sec. II. In what follows, their relationship to common local structural indicators such as free-volume, energy, stress, and frustration will be investigated.

\section{Structural features of SU(2) bond environments and their medium range connectivity \\ 1. Free volume}

Free volume content is a glassy material parameter that can be used to characterize the degree to which an amorphous structure is relaxed, and also how it might respond to an external load. Early theoretical work by Cohen and Turnbull [52] and Spaepen [53] viewed free volume as well-localized vacancy-type defects, whereas subsequent work views it as a "diffuse" delocalized quantity underlying variations in local atomic density [54,55]. Structures with lower free volume (higher densities) tend to be more relaxed. In what follows, a local free volume measure is loosely defined as a deviation of local atomic volume from a reference volume that is presently not defined. In other words, a reduction in local atomic volume would correspond to a decrease in free volume. To assign a volume to a particular atom is somewhat arbitrary, and usually involves either a Voronoi or radical Voronoi tesselation-or in the present work a modified radical Voronoi tesselation.

Structurally, regions with high icosahedral content generally contain decreased free volume content. This correlation turns out to be linear suggesting the reduction of free volume and the creation of icosahedral content are one and the same thing [56]. Whilst free volume does not strongly correlate with the spatial occurrence of stress driven athermal structural instabilities (STZ) [57], regions of reduced free volume tend to exhibit reduced activity involving the thermal activation of localized structural excitations $[56,58]$.

Figure 5 plots the average atomic volume for the relevant bond topologies, using the modified radical Voronoi tessellation. Inspection of the figure reveals the well known result that the $(0,12,0)$ topology, the icosahedrally coordinated environment, has the overall lowest volume for each atom type [56], being well below the global average. The figure also reveals that for each class of bond topologies (distinguished by the number of fourfold bonds) it is the topology with the lowest number of sixfold bonds that has the lowest average volume. As the number of sixfold bonds increases, so does the volume per atom, starting at values below the global average and ending at values above the global average. Inspection of the local atomic pressure [see Fig. SM 6(a)] demonstrates that regions with reduced volume (high density) are on average un-

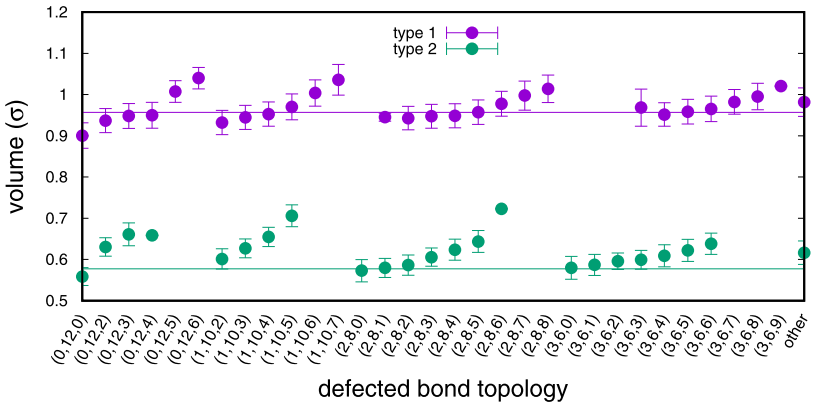

(a)

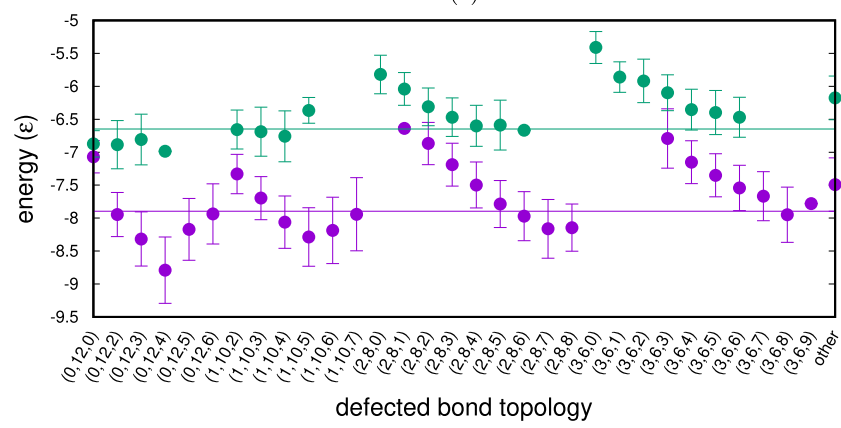

(b)

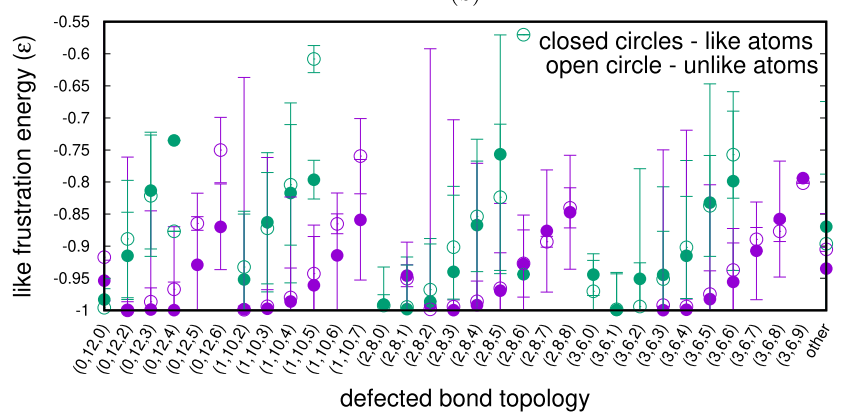

(c)

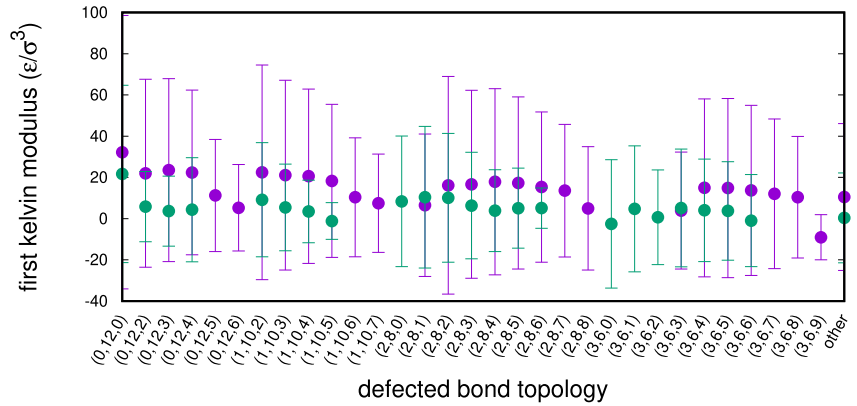

(d)

FIG. 5. Plot of average local (a) volume, (b) cohesive energy, (c) like/unlike atom frustration bond energy, and (d) Kelvin shear moduli for the local defected bond structures considered in Fig. 3. The lines in (a) indicate the average values for each atom type. In all figures, the error bars are derived from the standard deviation of the scatter. Data are derived from the $10-\mu$ s isotherm configuration.

der a negative (tensile) pressure, whereas those with enhanced volume (low-density regions) are on average under a positive (compressive) pressure. Thus their exists a strong spatial correlation between regions containing low numbers of defect bonds, and regions under tensile pressure which correspond to regions of higher than average density and therefore low free-volume content. 


\section{Local atomic energy}

The work of Ref. [9] found that increased icosahedral content also corresponds to a reduction in the average cohesive energy per atom. From the results of the previous section, one might therefore conclude that spatial regions containing a low bond defect density would also correspond to regions of low cohesive energy. Figure 5(c) shows the average energy per topology class for both atom types. Whilst this conclusion is certainly true of the $(0,12,0)$ environment for the smaller atoms, it is not the case for the larger atoms, where in fact the $(0,12,0)$ environment for the larger atom has one of the highest energies - a result compatible with the observation that the icosahedral content almost exclusively involves the smaller atom. The conclusion is also valid for the FrankKasper topologies for both atom types, where in general the local energy is less than the global average. Indeed, for both atom types, the lowest energy structure is not the bond-defect free topology $(0,12,0)$ but rather the Frank-Kasper Z16 coordinated topology of $(0,12,4)$. This observation most probably drives the nucleation of the Laves phase seen at higher temperatures [28] and the asymptotic nano-phase structure proposed in Ref. [9].

For the Nelson topologies, the trend is somewhat more complex. For both atom types, and for a given fourfold bond number, the energy is highest for the topology containing one sixfold bond and decreases with the addition of more sixfold bonds until a minimum is reached beyond which the energy again increases. For the smaller atom, those local environments containing one fourfold defect bond, the local cohesive energies are lower than the global average otherwise (for both atom types) it is those Nelson topologies with several sixfold bonds that are close to or below the global average cohesive energy value.

The understanding of these trends is difficult because the average atom-type of the nearest-neighbor population varies greatly between different realizations of actual atomic environments. See Figs. SM 6(b) and SM 6(c), which display the average number of like/unlike nearest neighbors, showing that it can vary greatly and systematically across the local topologies. It is therefore not an easy task to conclude via the cohesive energy of an atom, that a low-energy glassy structure corresponds to a low density of bond defects. To establish this expected correlation, the cohesive energy must be viewed in terms of bond frustration and the energy that it entails.

\section{Bond frustration}

Structural frustration involves the inability for bonds to mutually achieve the minimum energy (at the equilibrium bond length). Defining the fractional deviation away from this equilibrium bond length as $f_{a b}$ and the corresponding energy as $V_{a b}=V_{a b}\left(r\left(f_{a b}\right)\right)$ (see Sec. SM 7), the average geometrical frustration of like and unlike atoms may be calculated for both atom types, as a function of defect bond topology. These average geometrical frustration values may then be inserted into $V_{a b}$ to calculate the corresponding energy of frustration. Figure 5(c) does this and directly shows that for both atom types, those local topologies with a minimum number of bond defects generally have the lowest energy of frustration. Where this is sometimes not the case, is in the regime when the number of fourfold bonds is greater than or equal to the number of sixfold bonds [for example, the $(2,8,1)$ for the larger atom and the $(3,6,0)$ for the smaller atoms]. Because of their low coordination, such environments are however quite rare $(\lesssim 0.2 \%$ of the identified topologies).

Figure 5(c) establishes numerically that a glassy structure with a reduced bond-defect content does indeed correspond to the minimally frustrated low-energy structure. To connect these data to the local cohesive energy averages shown in Fig. 5(b), an estimate of the total energy using the frustration energy entailed in Fig. 5(c) and the average nearest-neighbor occupancies [see Figs. SM 6(b) and SM 6(c)] can be made. This estimate of the cohesive energy is shown in Fig. SM 6(d) and is qualitatively similar to that of the actual local cohesive energy averages calculated using the entire range of the Lennard-Jones interaction [Fig. 5(b)]. Indeed, detailed comparison indicates that the effect of more distant interactions is mainly characterized by a global energy offset, suggesting that these contribute to the energetics in mean-field-like way. This result demonstrates explicitly the notion that the short-range repulsive part of the interaction is the controlling interaction and the origin of the frustration [59-61].

\section{Local Kelvin elastic shear modulus}

A quantity that gives information on the local curvature of the PEL with respect to an affine distortion, may be obtained through the Born equation used to calculate the global elastic constants. In Sec. SM VIII, expressions are defined to obtain the local contributions to the global elastic constants. These may be seen as estimates of the local elastic stiffness moduli. Rather than expressing the elastic constants in the familiar Voigt notation, the Kelvin [62] notation is used. This is also a matrix of rank six, but retains its tensor structure giving meaningful eigenvalues equal to the elastic stiffness moduli with respect to the five linearly independent shear distortions and a single isotropic distortion. These eigenvalues are referred to as the five Kelvin elastic shear moduli and the dilation modulus. Figure SM 8 plots the distribution of the sorted local shear moduli for the $10-\mu$ sec sample. The distributions show that the lowest Kelvin shear modulus can be negative implying that the corresponding shear distortion (given by the corresponding eigenvector) results in a reduction in energy of the distorted bonds of the atom's neighbors. This does not imply a local material instability since the atomic environment beyond the central atom and its bonded neighbors stabilizes the local structure. The work of Ref. [63] hypothesized that these elastically "soft" regions are amenable to nearby low barrier energies within the PEL.

Figure 5(d) displays the atom-resolved average lowest Kelvin elastic modulus for each of the SU(2) topologies. Inspection of the actual distribution of moduli for each topology reveals a lower average first Kelvin modulus corresponds to a distribution whose low-end tail extends further into the negative region. Figure 5(d) demonstrates the local shear stiffness is generally softer for the smaller atom. The stiffest environment is that of an icosahedrally coordinated large atom. This however occurs rarely. The more common small atom icosahedral and large atom Frank-Kasper environments have the next highest values. For both atom types, the lowest Kelvin 


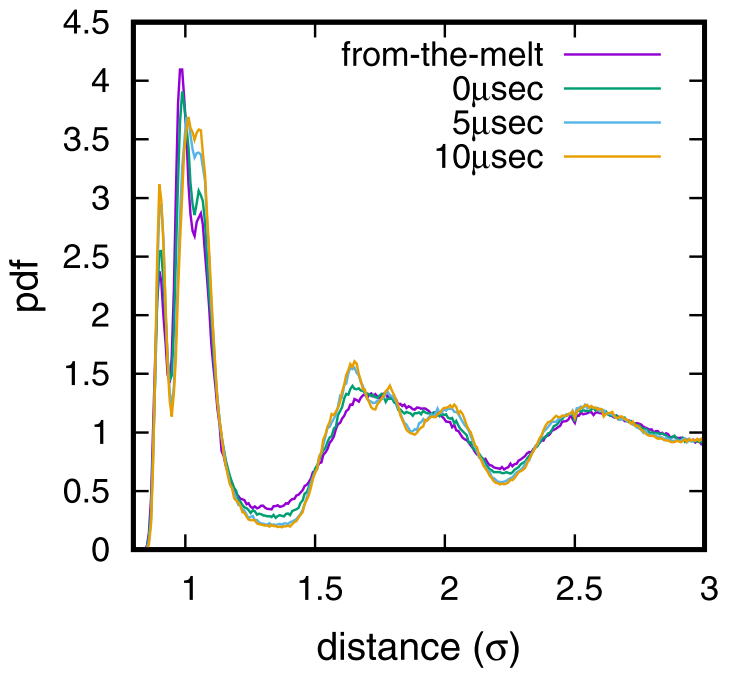

FIG. 6. Evolution of pair distribution as a function of structural relaxation.

shear moduli of the Nelson topologies $\left(1,10, N_{6}\right),\left(2,8, N_{6}\right)$, and $\left(3,8, N_{6}\right)$, generally decreases for increasing $N_{6}$, eventually being close to zero or negative for the smaller atom. Atomic environments identified as "other" have among the lowest shear moduli. The usefulness of this local measure will be discussed in detail in Sec. IV B.

\section{Medium range order}

The pair distribution function (PDF) gives some insight into the short and medium range atomic structure of a glass and is indirectly accessible via $\mathrm{x}$-ray diffraction. It is generally characterized by a strong peak at distances comparable to the nearest-neighbor separation between atoms, showing that like that of the liquid, the glass has strong short range order. At larger distances comparable to the second-nearest-neighbor distances, a very broad peak is seen which (for well relaxed glasses) has some structure indicating medium range order (MRO). Such MRO is difficult to see in atomistic simulation due to the short timescales simulated, however the present micro-second scale simulation do result in a sufficiently relaxed amorphous structure to reveal this MRO signature [9].

Figure 6 plots the total PDF for the four glass samples considered in the present work. As a function of degree-ofrelaxation, the short range structure (first peak) evolves and a medium range (second broad peak) develops. This latter fine-structure is entirely absent in the from-the-melt configuration. Sec. SM IX presents an investigation of the SU(2) topological classification origins of the MRO probed through the aforementioned PDF, and also the bond angle distribution function and icosahedral orientation correlation function developed by Nelson and co-workers $[30,64,65]$. It finds that the MRO of this model glass is characterized by fragments of bond networks whose geometry is similar to the polyhedral backbone of the C15 Laves structure. It is noted that the structure of the C15 Laves crystal is characterized by an extended array of sixfold defect bonds mediated by a connected network of Frank-Kasper $(0,12,4)$ local geometries. This is interpenetrated by a network of defect free bonds. For the case of our glass, fragments of this type will require a population of $(0,12,2)$ and $(0,12,3)$ local geometries to accommodate the disorder. This originates from two distinct contributions, that of fivefold bond fragments underlying the icosahedral defect free structures and that of sixfold bonds underlying FrankKasper defect lines characterized by the $(0,12,2),(0,12,3)$, $(0,12,4)$, etc. local topological environments. Bond connectivity between these two networks is via fourfold bond defect lines whose termination is described by the Nelson polyhedra and the rules of the SU(2) algebra. This later environment appears more random due to the large configurational possibilities of the fivefold and sixfold fragments of the C15 structure. These observations are entirely compatible with population histograms shown in Fig. 3 and demonstrate the more general picture given by the $\mathrm{SU}(2)$ formalism.

\section{Atomic and disclination visualization}

As pointed out in Sec. II, the defect bonds may be viewed as a disclination along which the icosahedral rotational symmetry is broken. Thus an atomic structure described by defect bonds can be seen as a network of disclination lines. The present section will investigate the properties of this disclination network as a function of glass structure relaxation. An initial overview of how such a defect structure evolves is gained via an inspection of the defect free bonded regions, which consist of the fivefold bonds and in particular the $(0,12,0)$ icosahedral environments. Figure SM 10 visualizes only the icosahedral regions of our four glass configurations, in which like-colored atoms belong to the same bond-connected cluster. From the melt, only small clusters of icosahedrally coordinated atoms exist. However upon isothermal relaxation, these grow and coalesce, forming a system spanning cluster of icosahedral atoms involving about $30 \%$ of the atoms. This already occurs within the first microsecond of the simulation. Section SN X displays [Fig. SN 11(a)] and discusses the corresponding icosahedral cluster distributions.

The $\mathrm{SU}(2)$ approach now provides a more detailed explanation of these trends. Figure 7(a) and 7(b) show the initial from-the-melt and $10-\mu \mathrm{sec}$ annealed configurations, respectively, visualized in terms of bonds for which the four-, five-, and sixfold bonds are colored according to the usual green, blue, and red convention of Figs. 1 and 4. Inspection of the two figures reveals an increase in blue (defect free) bonds associated with the increase of $(0,12,0)$ content. Indeed, there exist larger regions of defect free bonds in (b) which can be associated with the system spanning cluster of icosahedral environments. Inspection of both (a) and (b) reveal that within these regions red (sixfold) bonds are mainly seen, indicating that sixfold disclinations tend to penetrate the defect-free regions, whereas the fourfold disclinations tend to be located around these regions. This may be more easily seen in the insets displayed in (a) and (b) corresponding to smaller regions in which the defect free bonds have been removed to reveal the local disclination network of four- and sixfold bonds. Closer inspection of the sixfold disclination network extending into defect free environments of the more relaxed sample [Fig. 7(d)] reveals the Frank-Kasper topologies of $(0,12,2),(0,12,3),(0,12,4)$ - connected fragments of the Laves C15 backbone polyhedra-whereas a network 
(a)
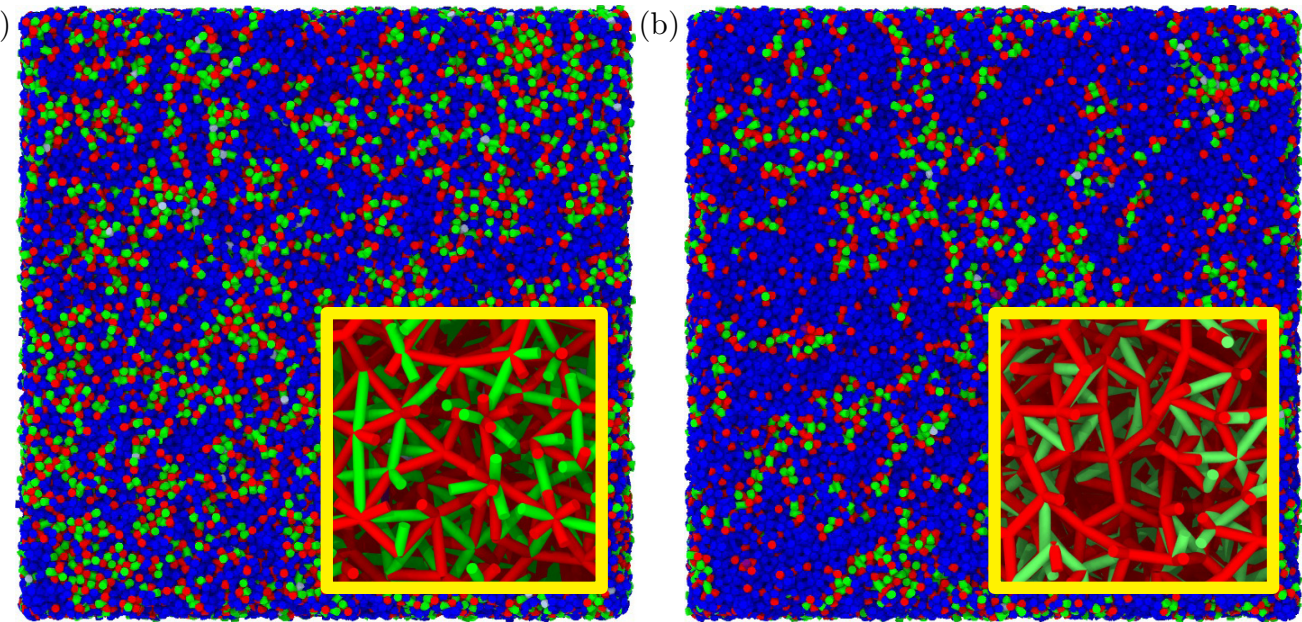

FIG. 7. Visualization of bonds colored according to their $n$-fold value (4-green, 5-blue, 6-red) for the (a) from-the-melt and (b) 10- $\mu$ sec configurations. Insets display a region showing only defected bonds and the local fourfold and sixfold disclination line network.

of fourfold and sixfold bonds exist at the boundaries of these defect regions. On the other hand, for the from-the-melt sample which contains only small (but numerous) regions of predominantly defect-free bonds, the extent of the regions dominated by Frank-Kasper topologies is reduced and a more homogeneous distribution of fourfold and sixfold bonds exists throughout the structure.

The connectivity of the disclination network is characterized by large clusters of atoms connected by bonds of the same order. For all samples, all atoms are connected via the defectfree fivefold bonds. On the other hand for the from-the-melt sample there exists one cluster of 29049 atoms connected by sixfold bonds, with the remaining atoms not being connected by sixfold bonds. For the fourfold bonds, there exists one cluster of 26712 atoms connected by fourfold bonds, and then 483 clusters below sizes of 10 atoms, with all remaining atoms not being connected by fourfold bonds. Of these fourfold isolated atoms $69 \%$ are $(0,12,0)$ and $32 \%$ are Frank-Kaspar topologies which are mainly $(0,12,2)$ and $(0,12,3)$. Similar trends exist for the more relaxed structures, although the dominant cluster for the sixfold connected cluster has reduced to 22710 atoms whereas the for the fourfold connected cluster it has reduced to 12637 atoms with the smaller clusters numbering up 80 atoms. See Fig. SM 11(b) for the corresponding cluster size distributions for all four samples. Additionally, the Frank-Kaspar topologies of the fourfold isolated atoms now include the $(0,12,4)$ topology. These trends are compatible with increased content of defect-free bonds and the increased presence of the Frank-Kasper topologies.

Figure 7 should be compared to the schematic originally proposed by Nelson [29,30,51], which shows a defect network with a considerably lower line-defect density. In this schematic, so-called isolated bubble structures are shown consisting of two nearby $(1,12,2)$ topologies. Since both of these have a coordination of $Z=13$, and therefore share an extra atom, such a "bubble" defect was referred to as an intersitial defect. A similar structure involving nearby $(2,8,1)$ topologies was referred to as a vacancy defect. Such isolated structures, which should involve at least 4 atoms, where not observed in the atomic configurations of the current work.
The $(1,12,2)$ tended more to be at the boundary of between regions of defects and defect-free regions.

\section{DISCUSSION}

In 1952, Frank asked the question [40] "In how many different ways can one put twelve billiard balls in simultaneous contact with one, counting as different the arrangements which cannot be transformed into each other with out breaking contact with the center ball?". The answer is three, the two close-packed configurations of the face-centered cubic and hexagonal structures, and the icosahedron structure. For soft interatomic potential systems, this hard-sphere constraint entails a non-negligible barrier energy separates the three configurations, if the allowed transformation trajectories are constrained to a certain distance from the central atom. Relaxing this constraint by allowing the particles to move away from the central atom, can reduce this barrier energy. If these thirteen atoms are not alone, such as in a densely packed structure of a glass, this structural trajectory becomes limited, allowing one to conclude that generally the three configurations of Frank are separated by non-negligible energy barriers. A similar rational exists for the SU(2) defect structures of Nelson-energy barriers must be crossed for there to be a reorganization of the nearest-neighbor shell defect structure. Moreover, since this latter aspect involves topological defects within the structural degrees of freedom of the nearest-neighbor shell, the corresponding barrier energies are expected to be large. It is from this perspective that one must view the present formalism as a low-energy description of nearest-neighbor structure with the obvious caveat that it is an approximate theory.

\section{A. Material specific considerations}

How the present results may be affected by differing atomic size and chemical composition, and their relation to experimentally realizable glass systems, is now discussed. Insight into this question can be found in the work of Miracle and co-workers [16] who considered the efficient packing 
of the first nearest-neighbor shell of differently sized atoms. In particular they considered the most efficient packing of solvent atoms around a central solute atom as a function of $r_{\text {solute }} / r_{\text {solvent }}$ and found that depending on the value of this ratio, a particular choice of "surface" coordination was needed to better rationalize the known experimentally realizable binary glasses. They considered the regimes of $n=3,4$, 5,6 and higher surface coordinations which corresponds to the strain free efficient packing of the corresponding $n$-fold bonds. The $n=3,4$, and 5 surface coordinations were viewed as tetrahedral, octahedral and icosahedral arrangements of solvent atoms around a solute atom. The work found that specific values of $R=r_{\text {solute }} / r_{\text {solvent }}$ would optimally entail these quite different geometrical arrangements corresponding to respectively $0.225,0.414$ and 0.904 . For radii ratios between these values, a less efficient but geometrically similar packing was envisaged. Experimentally realizable binary glasses are mainly found with $R \approx 0.7-0.8$ and (less so) for values around $R=1.25$. Indeed, the frequency of glass occurrence as a function of $R$ tended to peak at specific values, reflecting an experimental tendency for optimal packing efficiency.

Historically, work on metallic glass production addressed the question of what minimum concentration of an alloying element (the solute atom) results in a glass. Values typically ranged between 5-20 atomic percent [66]. Modern metallic glasses exploit a much wider range of stoichiometries and at some concentration, the solute/solvent labeling must be interchanged-this is the so-called isostructural condition defined when the number of nearest-neighbor solute atoms is the same for both labeling choices [67]. For the present 50:50 configuration, the mean number of large nearest-neighbor atoms of a large atom is 6.6 and for the case of smaller atoms it is 5.3. These values change little as a function of degree of relaxed glassy structure.

The present work considers a system in which the atomic radii ratio is $r_{\text {small }} / r_{\text {large }}=5 / 6 \approx 0.83=(1.2)^{-1}$. Section SM XI discusses the two possible solute regimes and whilst close to the isostructural limit, the discussion suggests the smaller atom is the solvent atom at a 50:50 stoichiometry, giving this model binary system an $R=0.83$ which is typical of common experimentally realizable binary glasses of the AB alloys of $\mathrm{HfCu}, \mathrm{ZrCu}, \mathrm{PdSi}$ (also $\mathrm{CaAl}$ with $R=0.7$ ) with $\mathrm{B}$ being the solute atom. The corresponding isostructural concentrations are in the range $42 \%-52 \%$. An increase in the smaller atom concentration should transition the system to the BA alloy analog and an $R=1.2$, with the larger atom now being the solute atom. The common experimentally realizable alloys are now the $\mathrm{CuZr}$ and $\mathrm{CuHf}$ (and $\mathrm{CaAl}$ with $R=1.116$ ).

The above results demonstrate a compatibility with the glass structural model of Ref. [16] and suggest that changing the values of $r_{\text {small }} / r_{\text {large }}$ will change the populations of four-, five-, and sixfold bonds, and thus the icosahedral population within the binary glass structure. Moreover in the extreme limit of this ratio becoming very small or very large, the present description (in terms of the fivefold symmetry of the icosahedron) might break down due to the presence of threefold and sevenfold bonds. Such regimes of radii ratios are however not common experimentally since the minimum concentrations needed for glass formation can be up to $10 \%-$
$20 \%$. The work of Ref. [16] has been extended to overlapping nearest-neighbor shells, producing the so-called efficient cluster packing model of glass structure and a theory of MRO [17-19]. It is an interesting prospect that the present SU(2) theory of bond-defects might facilitate a more precise realization of such packing models.

Returning to the work of Ref. [32] who investigated the $\left(N_{4}, N_{5}, N_{6}\right)$ topologies for a $\mathrm{Mg}_{3} \mathrm{Ca}_{7}$ alloy system. For this system, $r_{\mathrm{Mg}} / r_{\mathrm{Ca}} \approx 0.83=(1.2)^{-1}$ suggesting it should behave similarly to that of the present system when at the same stoichiometry. Figure SM 12 compares the published populations $\left(N_{4}, N_{5}, N_{6}\right)$ to the present $0 \mu$ sec configuration using a semilogarithmic plot. It shows that despite $89 \%$ of the atoms being identified as "other", the qualitative structure of the populations remains similar. The $(0,12,0)$ population is similar to the from-the-melt sample, suggesting the low populations of other $\mathrm{SU}(2)$ environments in the $\mathrm{Mg}_{3} \mathrm{Ca}_{7}$ data are not due to a lack of relaxation. It is noted that for a 30:70 stoichiometry, the present Lennard Jones potential entails a structure filled with defected close-packed structures [9] which would significantly reduce the SU(2) content. Thus differences seen in this past work might ultimately be due to the different stoichiometry regime considered. Finally, Fig. SM 12 also includes the SU(2) population for a 50:50 CuZr simulation using the embedded atom potential by Mendelev and co-workers [68] derived from an analogous preparation protocol corresponding to the present $0-\mu$ sec annealed sample. Again, apart from the absolute magnitudes, similar trends are seen across the Frank-Kasper and Nelson classes.

That the atomic size ratio can give such remarkable insight into metallic glass structure is a result of the unsaturated isotropic bonding between the metal atoms, suggesting a rather broad application for the SU(2) disclination description (as evidenced in Fig. SM 12). Indeed, in Ref. [51], the expectation was that polydispersivity can be seen as a form of annealed disorder that renormalizes the level of geometrical frustration. Nonisotropic interactions such as saturated bonding due to the presence of covalent elements and angular dependencies associated with strong d-state bonding in transition metals will strongly effect glass structure, possibly introducing new local structural motives that compete energetically with the low-energy motives of the SU(2) formalism. This distinction is also reflected in the different approaches in characterizing liquids and glasses that have nonisotropic and isotropic interaction [11] — the latter of which are focused more on tessellation based methods as done in the present work. For example, strong directional bonding can lead to network liquids and glasses that have quite different connectivity structures [69].

\section{B. Localized structural excitations (LSEs)}

The structural relaxation seen in the high temperature isothermal anneal is mediated by thermally activated LSEs [56,58,70,71]. Such LSEs also mediate stress relaxation [72] and the microplasticity occurring during the nominally elastic regime of deformation [73], and are also seen within the dynamical heterogeneities of the undercooled liquid [74-78] playing a central role in the change in atom mobility as the glass transition regime is entered [79]. 


\section{(a)}
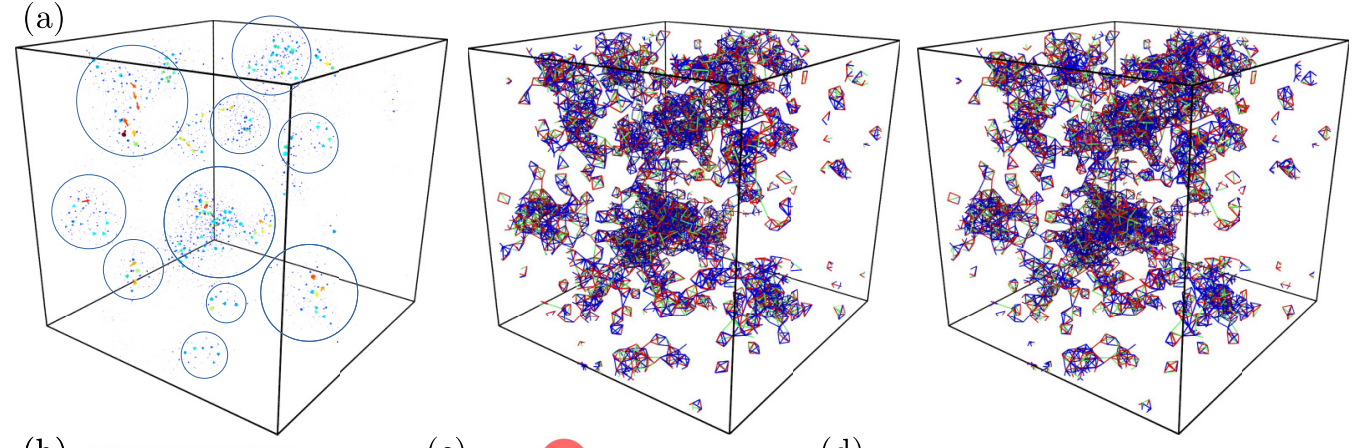

(b)

(c)
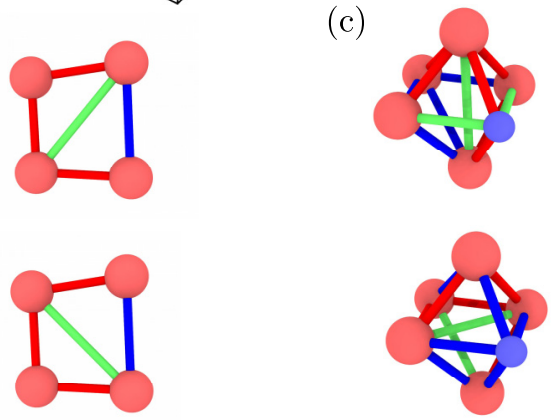

(d)

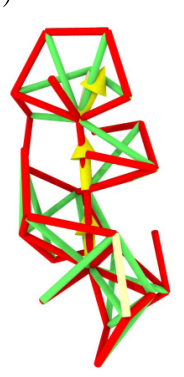

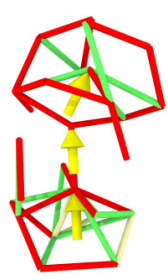

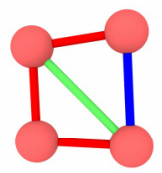

FIG. 8. (a) Visualization of atomic displacement field occurring between two configuration separated by 10 nanoseconds. Displacement vectors are colored red for displacement magnitudes comparable to the typical bond length and blue for displacement magnitudes associated with nonaffine strain. A number of localized structural excitations are circled, in which neighbor atoms successively displace. (b) and (c), Local structural motives whose bonding topologies change due to negligible atomic displacements. (d) Changes in bond topology due to bond-length displacements associated with a localized structural excitation.

Figure 8(a) visualizes an example of a number of thermally activated LSEs occurring between two configurations separated in time by 10 picoseconds at the 14 microsecond timescale of the isotherm anneal. The left-most panel plots the corresponding atomic displacement vectors, and colors them according to their magnitude. Red indicates the largest displacements, which are comparable to a bond length and represent the core structure of the LSE. This core structure generally involves bond-length scale displacements involving a few atoms, in which neighboring atoms replace each other's position forming a string/loop like displacement structure. The shorter blue displacement vectors indicate the smallest scale of displacement and generally correspond to the surrounding accommodating strain which accompanies the central LSE structure.

LSEs are driven by thermal fluctuations which provides the energy source to overcome a PEL energy barrier. Structural changes occurring via LSEs should be distinguished from the well known shear transformation zones (STZs) of Falk and co-workers [80], which arise via a stress driven inflection point in the PEL. Due to its athermal origin, STZ activity is inherently less stochastic than LSE activity, arising only because of an applied stress. A relevant question for both LSEs and STZs is, can the developed SU(2) description give insight into the local atomic environments more likely to undergo such activity?

It has already been established that quasi-localized lowfrequency vibrational modes of model glasses have a high oscillator strength in regions that are less-likely to contain icosahedral $(0,12,0)$ and Frank-Kasper $(0,12,4)$ topologies, and are more likely to exist in regions of so-called geometrically unfavoured motives (GUMs) [21] which generally involve the Nelson SU(2) topologies. Earlier work demonstrated that such vibrational "soft spots" where found to correlate strongly with regions exhibiting negative local Kelvin shear moduli which were hypothesized to more likely undergo structural rearrangement upon loading [63]. This was confirmed in the work of Ma and co-workers [21] which found that the GUMs had a propensity to mediate plasticity under high strain rate shear loading. This is compatible with other work, using the Wahnström potential, which found that local rigidity and therefore a resistance to shearing was intimately connected to local icosahedal content [81]. Work by Falk and co-workers [57] which exhaustively investigated what local features of the structure mediated stress-driven localized shear transformations, found little correlation with the traditional local structural indicators such as potential energy, density, the degree of short range order and local Voigt shear moduli. Whilst confirming the work of Ref. [21], they found the strongest correlation with a local yield shear stress measure which involved shearing only cluster of atoms extracted from their simulated glassy samples.

References [21,57] focus on predicting stress driven STZs and not the thermally activated LSEs shown in Fig. 8, which under a fixed applied strain geometry also mediate thermally activated shear stress relaxation [72] and microplasticity [73]. Indeed, it might be that predicting the location of an STZ and one prone to LSEs are quite different problems. The present work offers one way to study certainly the latter dynamics where the nature of the disclination network forms a predictive tool to understand both thermally activated structural relaxation and plasticity. 
Analysis of many configurations separated by 10 picoseconds show that the topologies $(0,12,6),(1,10,7),(2,8,8)$, $(3,6,0),(3,6,2),(3,6,9)$, and "other" are mainly involved in LSE activity seen in Fig. 8(a). With the exception of $(3,6,0)$ and $(3,6,2)$, these topologies correspond to regions of larger volume [Fig. 5(a)], which is compatible with the observation that thermally activated LSEs are more likely to occur in regions of increased free-volume [9,56]. Figure 5(d) also reveals these topologies have a low average first Kelvin shear modulus indeed suggesting a link between so-called "soft spots" and low barrier energies. In fact, a strong indicator of this link is the observation that the smaller atom is generally in elastically softer environments and also the most thermally active in terms of general LSE mediated mobility [58]. The topologies of $(3,6,0)$ and $(3,6,2)$ correspond to undercoordinated environments which generally entails a lower free-volume. They also have a low first Kelvin modulus further suggesting a connection between stiffness and barrier energy that does not involve enhanced free volume. From Fig. 3, these two topologies are rare, indicating that when they do exist, they are likely to transform into other local topologies via the thermal activation of an LSE.

The picture which therefore emerges is that the Nelson topologies with large values of $N_{6}$ or regions not describable by the SU(2) framework are more likely to facilitate thermally activated LSE activity. These topologies on average entail a high disclination defect density characterized by high bond frustration resulting in high free volume and low Kelvin elastic stiffness.

\section{LSE activity and changes in local SU(2) topology}

The LSE structures seen in the left-most panel of Fig. 8(a) entail a change in SU(2) topology. This is shown in the center and right most panels of the figure, which visualizes only the change in the $n$-fold bond structure. Here, only atoms whose local topology has changed are displayed. The bond structure (at the start and end) connecting these atoms is also displayed. A broad inspection of the spatial structure shows strong overlap with the identified LSEs of the left panel-an obvious result reflecting the strong change in the local bonding environment due to the displacements associated with the LSEs.

In regions away from the LSEs, where there only exist small displacement fields due to the accommodating strain fields of the LSEs, changes in the local bonding topology are largely absent. In these parts of the structure, there do however exist localized and isolated changes in the bonding topology. Figures 8(b) and 8(c) display a zoom-in of some common examples of such changes. Here the upper and lower panel of (b) and (c) show the configurations at the start and end of the 10 picosecond interval. In (b), which displays a planar structure, a fourfold bond has switched between two different pairs of atoms with no other change in bond structure between the displayed atoms. On the other hand, (c) which displays a sextet of atoms associated with a fourfold bond indicates a switching of this fourfold bond between two different atom pairs. In this case, the switching results in a different bonding structure between the surrounding common-neighbor atoms.
Given that the above examples occur in regions experiencing minor nonaffine displacements associated with surrounding accommodating strain of the LSE, one might simply attribute such fluctuations to the chosen criterion of what constitutes a nearest-neighbor bond. This is a generic problem of metallic systems due to the de-localized nature of the atomic bonding, relegating the choice of atom size and bonding connectivity to a nonunique choice of local geometry such as in the Voronoi and radical-Voronoi tessellations. Alternatively, this ambiguity can be seen to reflect a type of degeneracy in the topology which also corresponds to an obvious approximate degeneracy in the bond frustration energy (see Sec. SM VII).

In regions where LSEs occur there exist strong changes in the nearest-neighbor structure and therefore in the $n$-fold bonding structure. Fig. 8 d gives one example of this where the LSE is characterized by three atoms linearly displacing (indicated by the yellow arrows). Surrounding this LSE is a displacement field that reduces in magnitude as the distance from the central LSE increases (not shown). The left and right panels in d) show the non-five-fold structure associated with the nearest neighbors of the three-atom LSE, at the start and end of the 10 picosecond interval. The local structure of the lower atom contains non-four- and non-six-fold bonds, and therefore is in an environment not straight-forwardly characterized by the SU(2) topology of Sec. II. On the other hand, the central atom transits from a Nelson $(2,8,5)$ topology to a Frank-Kasper $(0,12,3)$ topology, whereas the upper atom whose initial four- and sixfold environment could not be uniquely identified has transited to the Nelson $(2,8,2)$ topology. Thus the LSE has resulted in a reduction of the local defect structure and the creation of a Frank-Kasper sixfold structure.

More generally, the above analysis suggests a topological reason for the observed string-like geometry of the LSEsany change in the local disclination structure must be to some extent nonlocal and one-dimensional-like, since it will involve a reorganization of line-defects that at each site must satisfy the SU(2) local topology.

Finally, since thermally activated LSE activity mediates structural relaxation, it also mediates the creation of increasing $(0,12,0)$ content. The most likely initial local topologies where this transformation occurs are the $(1,10,2),(2,8,2)$, $(2,8,4),(3,6,4)$, and "other" environments. When one determines the actual number of four- and sixfold bonds involved in the observed transformation to the $(0,12,0)$ topology, a roughly equal number of four- and sixfold bonds contribute, which reflects the $n$-fold bond population gradients with respect to an increase in icosahedral content investigated in Sec. III A. Figure 5(a) reveals these to have relatively low local volumes (reduced free volume content) and intermediate first Kelvin elastic moduli, suggesting that such transformations are less likely to occur. Thus as the system relaxes, and the bond defect network evolves to a less frustrated configuration, the density of free-volume reduces with a corresponding increase in Kelvin shear stiffness, and LSEs leading to the creation of icosahedral content become less frequent resulting in a slowing down of the relaxation process (as seen in Figs. 2 and SM 1a). 


\section{Correlated disorder and course grained theories of the structural glass}

The SU(2) requirement introduces a local bonding constraint that, if satisfied everywhere, will lead to a disclination structure characterizing the frustration and structural disorder of the glass. In this sense, glasses may be seen as containing correlated disorder. Correlated disorder also exists in magnetism where for example in spin-ice local rules constrain the orientation of neigboring spins $[82,83]$. This constraint entails no-long range magnetic order and is referred to as cooperative paramagnetism or a spin glass state, leading to a coarse-grained divergenceless magnetic field, the breaking of which, introduces charged magnetic monopoles and an emergent dynamics analogous to electromagnetism [84].

There also exist attempts to derive a course grained theory of glasses based on the SU(2) local constraint presently investigated [30,51,85-87]. These have been developed within the framework of Landau's particle-density free energy formalism of liquid-to-solid freezing [88], which involves minimizing a generalized (Ginzburg) gradient term proportional to $\left.\mid D_{\mu} \delta \rho_{n}(\mathbf{r})\right)\left.\right|^{2}$. Here $D_{\mu}$ has a similar structure to a covariant derivative and $\delta \rho_{n}(\mathbf{r})$ - the order parameter-is a coarse grained density fluctuation away from the global value characterized through a symmetry labeled by $n$. In addition to the gradient term of the free energy, there exist quadratic and cubic terms with respect to $\delta \rho_{n}(\mathbf{r})$, the latter of which indicates a first order freezing transition [88].

For equilibrium freezing to a close-packed polycrystalline solid, $n$ represents a reciprocal lattice vector magnitude reflecting the material's translational and rotational symmetry. In this case the free energy term involving $D_{\mu}$ can be minimized to zero every where, resulting in any two infinitesimally spaced material locations being related by the generator of the associated symmetry. For the undercooled liquid an appropriate $n$ will represent a point-group symmetry such as that of the icosahedron. The inability to pack undistorted tetrahedra in a space filling way in three dimensions makes the minimization of $\left.\mid D_{\mu} \delta \rho_{n}(\mathbf{r})\right)\left.\right|^{2}$ to zero, everywhere, impossible. Fundamentally this is due to the non-Albelian nature of the associated symmetry generators, manifesting itself as line defects (disclinations) along which the icosahedral symmetry is broken to accommodate this noncommutivity.

The above approach has motivated the conjecture that the metastable solid in which the undercooled liquid freezes is that of a Frank-Kaspar crystal consisting of an ordered network of disclinations. Indeed, as the temperature drops below the equilibrium melting temperature, $T_{\mathrm{m}}$, the undercooled liquid will transit to a Frank-Kasper polycrystal at some lower critical temperature $T_{\mathrm{c}}$, and that a glass is created when the system falls out of metaequilibrium before this temperature is reached entering the new glassy metaequilibrium. The structural evolution of the glass then becomes arrested due to the entanglement of the disordered frozen-in disclination network.

The recent interest in the so-called G-glass seen in atomistic simulation of the $\mathrm{Ag}$ and $\mathrm{CuAg}$ systems [10,89], and the experimental observation of its first-order character in the ternary glass $\mathrm{PtCuPh}$ [90], constitutes an example of a possibly uniquely metaequilibrium phase transition occurring in the undercooled liquid regime. However in the case of the simulations, the resulting crystallinity is predominantly of a close-packed nature [10] and thus most likely not topologically distinct from the liquid-to-solid phase transition existing under equilibrium conditions.

Efforts to develop a quantitative theoretical understanding of the glass transition have focused on a change from homogeneous to heterogeneous spatiotemporal dynamics [79] that can be captured via coarse grained kinetically constrained lattice models [61,91]. Central to this transition are the stringlike excitations that mediate this emerging heterogeneous mobility - a dynamics that is referred to as being both facilitated and hierarchical in which atoms undergo temporal and spatial intermittent motion. Within this framework a dynamical first-order phase transition emerges associated with an order parameter that measures this dynamical facilitation. Theoretically, this is described via an out-of-equilibrium statistical mechanics involving the enumeration of corresponding trajectories rather than atomic configurations as in equilibrium statistical mechanics [79].

These considerations and the results of the present work motivate the conclusion that, as the temperature of the undercooled liquid decreases there exist regions of increasing temporal and spatial extent that largely satisfy the SU(2) constraints. The present work has only analysed the inherent state structures arising from cg quenching, but one can equally well study instantaneous configurations from atomistic simulations of the glass transition, as has been done in Ref. [13] which investigated the statistics of local structural motives for the Wahnström potential. Using the SU(2) formalism, their connectivity or equivalently the spatial extent of the fluctuating disclination network may be studied. Indeed, both the spacetime "bubbles" of inactivity (regions of low frustration and minimal disclination content) and the stringlike excitations (LSEs) that drive the heterogeneous dynamics, should be intimately related to the constraints associated with the SU(2) formalism. Interestingly, whereas the $\mathrm{SU}(2)$ regions might impose hard constraints on allowed LSEs, the role of environments not satisfying the SU(2) picture, which the present work indicates to be more LSE active, might also play an important role in the facilitation, just as the imposition of soft constraints has in kinetic facilitation [91]. Finally, via the statistics of the local minima of the course grained free energy landscape formalism $[51,86]$, a connection could be made between the spatial fluctuations in $\delta \rho_{n}(\mathbf{r})$ and that of the mobility/activity order parameter of these kinetically constrained facilitation models $[61,79,91]$.

\section{CONCLUDING REMARKS}

A quantitative understanding of the nature of the structural constraints of a well-relaxed amorphous solid could play just as an important role as that of the theory of defects in a crystalline system. For the crystal, the reference is the long-range order of the perfect lattice, whereas for a glass, the reference is most likely a variety of low-energy structural motives obeying the aforementioned constraints. These very same constraints also define the nature of the local structural excitations that will mediate glassy material evolution and its response to 
external stimuli as a load. The present work demonstrates that the early work of Frank, Kasper, Bernel, Turnbull, and Chaudri culminating in the work of Nelson, whose mathematically rigorous description of defects in the first neighbor shell forms a consistent and possibly robust (albeit approximate) theory of bond defects within the amorphous solid. The present work finds that the vast majority of well relaxed structure have local bonding topologies that follow the prediction of the associated SU(2) algebra developed in Ref. [30], both in terms of $n$-fold bond populations and also in terms of the predicted orientational geometry of the bonds. This results in a dense and extended disclination line-defect structure embedded in a system spanning network of icosahedrally coordinated atomic environments. This defect network is found to exhibit a spatial icosahedral orientational correlation extending up to 3 to 4 bond lengths. Within this context the work has numerically demonstrated that a less frustrated defect network (as defined by deviations away from equilibrium bond-lengths) will correspond to a less dense network of defect lines and therefore an increased icosahedral content and a more relaxed glassy structure. The study of atoms of different size ratios and how the addition of larger atoms affects this description forms the natural next steps to multicompound alloy mixtures which form the industrially relevant bulk metallic glasses. More generally, the provided SU(2) visualization tools [34] will motivate future structural analysis of the undercooled, the glass transition and glass structure in terms of structural motive connectivity.

The revelation that most of our glassy structure does indeed satisfy a set of local mathematically well-defined constraints, which in turn result in an emergent longer range correlation, suggests a certain level of correlated disorder. As with point, line and planar defects in crystals, the exploitation of such a disorder context, can lead to detailed theories of the glass transition, structural evolution involving aging and rejuvenation, and ultimately that of a thermally activated theory of plasticity.

\section{ACKNOWLEDGMENTS}

The present work was supported by the Swiss National Science Foundation under Grant No. 200021-165527. The author thanks W. L. Johnson, R. Maass, and D. Rodney for useful discussions.
[1] W. Kauzmann, The nature of the glassy state and the behavior of liquids at low temperatures, Chem. Rev. 43, 216 (1948).

[2] H. Sillescu, Heterogeneity at the glass transition: A review, J. Non-Cryst Sol. 243, 81 (1999).

[3] M. D. Ediger and P. Harrowell, Perspective: Supercooled liquids and glasses, J. Chem. Phys 137, 080901 (2012).

[4] M. Goldstein, Viscous Liquids and the Glass Transition: A Potential Energy Barrier Picture, J. Chem. Phys. 51, 3728 (1969).

[5] P. G. Debenedetti and F. H. Stillinger, Supercooled liquids and the glass transition, Nature (London) 410, 259 (2001).

[6] P. Chaudhari and D. Turnbull, Structure and propertues of metallic glasses, Science 199, 11 (1978).

[7] T. C. Hufnagel, C. A. Schuh, and M. L. Falk, Deformation of metallic glasses: recent developments in theory, simulations, and experiments, Acta Mater. 109, 375 (2016).

[8] Y. Sun, A. Concustell, and A. L. Greer, Thermomechanical processing of metallic glasses: Extending the range of the glassy state, Nat. Rev. Mater. 1, 16039 (2016).

[9] P. M. Derlet and R. Maass, Emergent structural length scales in a model binary glass - the micro-second molecular dynamics time-scale regime, J. Alloys Comp. 821, 153209 (2020).

[10] Q. An, W. L. Johnson, K. Samwer, S. Y. L. Corona, and W. A. Goddard, III, Formation of two glass phases in binary $\mathrm{Cu}-\mathrm{Ag}$ liquid, Acta Mater. 195, 274 (2020).

[11] H. Tanaka, H. Tong, R. Shi, and J. Russo, Revealing key structural featureshidden in liquids and glasses, Nat. Rev. Phys. 1, 333 (2019).

[12] J. P. Straley, Crystallization in curved three-dimensional space, Phys. Rev. B 30, 6592 (1984).

[13] F. Turci, G. Tarjus, and C. P. Royall, From Glass Formation to Icosahedral Ordering by Curving Three-Dimensional Space, Phys. Rev. Lett. 118, 215501 (2017).
[14] F. C. Frank and J. S. Kasper, Complex alloy structures regarded as sphere packings. I. Definitions and basic principles, Acta Crystallogr. 11, 184 (1958).

[15] J. D. Bernal, Proc. R. Soc. London Ser. A 280, 299 (1964).

[16] D. B. Miracle, W. S. Sanders, and O. N. Senkov, The influence of efficient atomic packing on the constitution of metallic glasses, Phil. Mag. 83, 2409 (2003).

[17] D. B. Miracle, A structural model for metallic glasses, Nat. Mater. 3, 697 (2004).

[18] D. B. Miracle, The efficient cluster packing model—An atomic structural model for metallic glasses, Acta Mater. 54, 4317 (2006).

[19] K. J. Laws, D. B. Miracle, and M. Ferry, A predictive structural model for bulk metallic glasses, Nat. Commun. 6, 8123 (2015).

[20] H. W. Sheng, W. K. Luo, F. M. Alamgir, J. M. Bai, and E. Ma, Atomic packing and short-to-medium-range order in metallic glasses, Nature (London) 439, 419 (2006).

[21] J. Ding, S. Patinet, M. L. Falk, Y. Cheng, and E. Ma, Soft spots and their structural signature in a metallic glass, Proc. Natl. Acad. Sci. USA 11, 14052 (2014).

[22] E. Ma, Tuning order into disorder, Nat. Mater. 14, 547 (2015).

[23] H. Jónsson and H. C. Andersen, Icosahedral Ordering in the Lennard-Jones Liquid and Glass, Phys. Rev. Lett. 60, 2295 (1988).

[24] J. Zemp, M. Celino, B. Schönfeld, and J. F. Löffler, Icosahedral superclusters in $\mathrm{Cu}_{64} \mathrm{Zr}_{36}$ metallic glass, Phys. Rev. B. 90, 144108 (2014).

[25] J. Zemp, M. Celino, B. Schönfeld, and J. F. Löffler, Crystal-Like Rearrangements of Icosahedra in Simulated Copper-Zirconium Metallic Glasses and Their Effect on Mechanical Properties, Phys. Rev. Lett. 115, 165501 (2015). 
[26] G. Wahnström, Molecular-dynamics study of a supercooled two-component Lennard-Jones system, Phys. Rev. A 44, 3752 (1991).

[27] U. R. Pedersen, T. B. Schrøder, J. C. Dyre, and P. Harrowell, Geometry of Slow Structural Fluctuations in a Supercooled Binary Alloy, Phys. Rev. Lett 104, 105701 (2010).

[28] U. R. Pedersen, N. P. Bailey, J. C. Dyre, and T. B. Schrøder, Crystallization of the Wahnström Binary Lennard-Jones Liquid, arXiv:0706.0813.

[29] D. R. Nelson, Liquids and Glasses in Spaces of Incommensurate Curvature, Phys. Rev. Lett. 50, 982 (1983).

[30] D. R. Nelson, Order, frustration, and defects in liquids and glasses, Phys. Rev. B 28, 5515 (1983).

[31] N. D. Mermin, The topological theory of defects in ordered media, Rev. Mod. Phys. 51, 591 (1979).

[32] D. W. Qi and S. Wang, Icosahedral order and defects in metallic liquids and glasses, Phys. Rev. B 44, 884 (1991).

[33] A. Stukowski, Visualization and analysis of atomistic simulation data with OVITOethe open visualization tool, Model. Simul. Mater. Sci. Eng. 18, 015012 (2010).

[34] See GitHub repository at https://github.com/derlet/SU2.

[35] C. H. Rycroft, Voro++: A three-dimensional Voronoi cell library in $\mathrm{C}++$, Chaos 19, 041111 (2009).

[36] See Supplemental Material at http://link.aps.org/supplemental/ 10.1103/PhysRevMaterials.4.125601 for additional information, analysis and data.

[37] G. M. Hocky, D. Coslovich, A. Ikeda, and D. Reichman, Correlation of Local Order with Particle Mobility in Supercooled Liquids is Highly System Dependent, Phys. Rev. Lett. 113, 157801 (2014).

[38] R. Pinney, T. B. Liverpool, and C. P. Royall, Recasting a model atomistic glassformer as a system of icosahedra, J. Chem. Phys. 143, 244507 (2015).

[39] C. P. Royall, A. Malins, A. J. Dunleavy, and R. Pinney, Strong geometric frustration in model glassformers, J. Non-Cryst. Solids 407, 34 (2015).

[40] C. Frank, Super cooling of liquids, Proc. R. Soc. A 215, 43 (1952).

[41] F. H. Stillinger and T. A. Weber, Hidden structure in liquids, Phys. Rev. A 25, 978 (1982).

[42] F. H. Stillinger and R. A. LaViolette, Local order in quenched states of simple atomic substances, Phys. Rev. B 34, 5136 (1986).

[43] S. Plimpton, Fast parallel algorithms for short-range molecular dynamics, J. Comput. Phys. 1, 119 (1995)

[44] G. Voronoi, Nouvelles applications des paramètres continus à la théorie des formes quadratiques. Primiere Mémoire: Sur quelques prepriétés des formes quadratiques positives parfaites, J. Reine Angew Math 133, 97 (1907).

[45] G. Voronoi, Nouvelles applications des paramètres continus à la théorie des formes quadratiques. Deuxième Mémoire: Recherches usr les parallíloédrès primitifs, J. Reine Angew Math 134, 198 (1908).

[46] B. N. Delaunay, Sur la sphèere vide. A la memoire de Georges Voronoi, Izv. Akad. Nauk SSSR Otd. Mat. i Estestv nauk 7, 793 (1934).

[47] B. J. Gellatly and J. L. Finney, Characterisation of models of multicomponent amorphous metals: The radical alternative to the Voronoi polyhedron, J. Non-Cryst. Solids 50, 313 (1982).
[48] J. D. Honeycutt and H. C. Andersen, Molecular dynamics study of melting and freezing of small Lennard-Jones clusters, J. Chem. Phys. 91, 4950 (1987).

[49] A. V. Anikeenko and N. N. Medvedev, Polytetrahedral Nature of the Dense Disordered Packings of Hard Spheres, Phys. Rev. Lett. 98, 235504 (2007).

[50] A. Malins, J. Eggers, C. P. Royall, S. R. Williams, and H. Tanaka, Identification of structure in condensed matter with the topological cluster classification, J. Chem. Phys. 138, 12A535 (2013).

[51] D. R. Nelson, Defects and Geometry in Condensed Matter Physics (Cambridge University Press, New York, 2002), Chap. 4.

[52] M. H. Cohen and D. Turnbull, Molecular transport in liquids and glasses, J. Chem. Phys. 31, 1164 (1959).

[53] F. Spaepen, A microscopic mechanism for steady state inhomogeneous flow in metallic glasses, Acta Metall. 25, 407 (1977).

[54] A. S. Argon, Plastic Deformation in Metallic Glasses, Acta Metall. 27, 47 (1979).

[55] T. Egami, Atomic level stresses, Prog. Mater. Sci. 56, 637 (2011).

[56] P. M. Derlet and R. Maass, Local volume as a robust structural measure and its connection to icosahedral content in a model binary amorphous system, Materialia 3, 97 (2018).

[57] S. Patinet, D. Vandembroucq, and M. L. Falk, Connecting Local Yield Stresses with Plastic Activity in Amorphous Solids, Phys. Rev. Lett. 117, 045501 (2016).

[58] P. M. Derlet and R. Maass, Thermal processing and enthalpy storage of an amorphous solid: A molecular dynamics study, J. Mat. Res. 32, 2668 (2017).

[59] B. Widom, Intermolecular forces and nature of liquid state, Science 157, 375 (1967).

[60] D. Chandler, J. D. Weeks, and H. C. Andersen, Van der Waals picture of liquids, solids, and phase transformations, Science 220, 787 (1983).

[61] J. P. Garrahan and D. Chandler, Coarse-grained microscopic model of glass formers, Proc. Natl. Acad. Sci. USA 100, 9710 (2003).

[62] W. Thomson, XX I. Elements of a mathematical theory of elasticity, Philos. Trans. R. Soc. Lond. 146, 481 (1856).

[63] P. M. Derlet and R. Maass, and J. F. Löffler, The Boson peak of model glass systems and its relation to atomic, structure, Eur. J. Phys. B 85, 148 (2012).

[64] P. J. Steinhardt, D. R. Nelson, and M. Ronchetti, Icosahedral Bond Orientational Order in Supercooled Liquids, Phys. Rev. Lett. 47, 1297 (1981).

[65] P. J. Steinhardt, D. R. Nelson, and M. Ronchetti, Bondorientational order in liquids and glasses, Phys. Rev. 8 28, 784 (1983).

[66] T. Egami and Y. Waseda, Atomic size effect on the formability of glasses, J. non-crystalline Solids 64, 113 (1984).

[67] D. B. Miracle, D. V. Louzguine-Luzgin, L. V. LouzguinaLuzgina, and A. Inoue, An assessment of binary metallic glasses: Correlations between structure, glass forming ability and stability, Int. Mater. Rev. 55, 218 (2010).

[68] M. I. Mendelev, M. J. Kramer, R. T. Ott, D. J. Sordelet, D. Yagodin, and P. Popel, Development of suitable interatomic potentials for simulation of liquid and amorphous $\mathrm{CuZr}$ alloys, Phil. Mag. 89, 967 (2009). 
[69] S. Roldán-Vargas, L. Rovigattic, and F. Sciortino, Connectivity, dynamics, and structure in a tetrahedral network liquid, Soft Matter 13, 514 (2017).

[70] S. Swayamjyoti, J. F. Löffler, and P. M. Derlet, Local structural excitations in model glasses, Phys. Rev. B 89, 224201 (2014).

[71] S. Swayamjyoti, J. F. Löffler, and P. M. Derlet, Local structural excitations in model glass systems under applied load, Phys. Rev. B 93, 144202 (2016).

[72] P. M. Derlet and R. Maass, Thermally-activated stress relaxation in a model amorphous solid and the formation of a system-spanning shear event, Acta Mater. 143, 205 (2018).

[73] P. M. Derlet and R. Maass, Microplasiticy in a model binary glass (unpublished).

[74] C. Donati, J. F. Douglas, W. Kob, S. J. Plimpton, P. H. Poole, and S. C. Glotzer, String-Like Cooperative Motion in a SuperCooled Liquid, Phys. Rev. Lett. 80, 2338 (1998).

[75] T. B. Schrøder, S. Sastry, J. C. Dyre, and S. C. Glotzer, Crossover to potential energy landscape dominated dynamics in a model glass-forming liquid, J. Chem. Phys. 112, 9834 (2000).

[76] Y. Gebremichael, M. Vogel, and S. C. Glotzer, Particle dynamics and the development of string-like motion in a simulated mono-atomic super-cooled liquid, J. Chem. Phys. 120, 4415 (2004).

[77] M. Vogel, B. Doliwa, A. Heuer, and S. C. Glotzer, Particle rearrangements during transitions between local minima of the potential energy landscape of a binary Lennard-Jones liquid, J. Chem. Phys. 120, 4404 (2004).

[78] T. Kawasaki and A. Onuki, Dynamics of thermal vibrational motions and string like jump motions in three-dimensional glass-forming liquids, J. Chem. Phys. 138, 12A514 (2013).

[79] D. Chandler and J. P. Garrahan, Dynamics on the way to forming glass: Bubblesin Space-Time, Annu. Rev. Phys. Chem. 61, $191(2010)$
[80] M. L. Falk and J. S. Langer, Dynamics of viscoplastic deformation in amorphous solids, Phy. Rev. E 57, 7192 (1998).

[81] R. Pinney, T. B. Liverpool, and C. P. Royall, Yielding of amodel glass former: An interpretation with an effective system of icosahedra, Phys. Rev E 97, 032609 (2018).

[82] C. Castelnovo, R. Moessner, and S. L. Sondhi, Magnetic monopoles in spin ice, Nature (London) 451, 42 (2008).

[83] L. Balents, Spin liquids in frustrated magnets, Nature (London) 464, 199 (2010).

[84] C. L. Henley, The "Coulomb phase" in frustrated systems, Annu. Rev. Condens. Matter Phys. 1, 179 (2010).

[85] D. R. Nelson and J. Toner, Bond-orientational order, dislocation loops, and melting of solids and smectic-A liquid crystals, Phys. Rev. B 24, 363 (1981).

[86] D. R. Nelson and M. Widom, Symmetry, Landau theory and polytope models of glass, Nucl. Phys. B 240, 113 (1984).

[87] A. Acharya and M. Widom, A microscopic continuum model for defect dynamics inmetallic glasses, J. Mech. Phys. Sol. 104, 1 (2017).

[88] On the theory of phase transitions, The Collected Papers of L. D. Landau, edited by D. ter Haar (Pergamon Pressm 1965), Chap. 29, p. 193.

[89] Q. An, W. L. Johnson, K. Samwer, S. L. Corona, and W. A. Goddard, III, First-order phasetransition in liquid Ag to the heterogeneous G-phase, J. Phys. Chem. Lett. 11, 632 (2020).

[90] J. H. Na, S. L. Corona, A. Hoff, W. L. Johnson, Observation of an apparent first-order glass transition in ultrafragile $\mathrm{Pt}-\mathrm{Cu}-\mathrm{P}$ bulk metallic glasses, Proc. Natl. Acad. Sci. USA 117, 2779 (2020).

[91] Y. S. Elmatad and R. L. Jack, Space-time phase transitions in the East model with a softened kinetic constraint, J. Chem. Phys. 138, 12A531 (2013). 